Elsevier Editorial System(tm) for Ocean Engineering

Manuscript Draft

Manuscript Number: OE-D-06-00042R1

Title: Internal Generation of Waves: Delta Source Function Method and Source Term Addition Method

Article Type: Full Length Article

Keywords: Numerical generation of wave; Internal generation of waves; Source term addition; Source function; Energy velocity approach; Boussinesq equations; Extended mild-slope equations; Numerical experiment

Corresponding Author: Professor Changhoon Lee, Ph.D.

Corresponding Author's Institution: Sejong University

First Author: Gunwoo Kim, Ph.D.

Order of Authors: Gunwoo Kim, Ph.D.; Changhoon Lee, Ph.D.; Kyung-Duck Suh, Ph.D.

Abstract: In this study, we investigate two internal wave generation methods in numerical modeling of timedependent equations for water wave propagation, i.e., delta source function method and source term addition method, the latter of which has been called the line source method in literatures. We derive delta source functions for the Boussinesq type equations and extended mild-slope equations. By applying the fractional step splitting method, we show that the delta source function method is equivalent to the source term addition method employing the energy velocity. This suggests that the energy velocity should be used rather than the phase velocity for the transport of incident wave energy in the source term addition method. Finally, the performance of the delta source function method is verified by accurately generating nonlinear cnoidal waves as well as linear waves for horizontally one-dimensional cases. 
Changhoon Lee, Ph.D.

Associate Professor

Dept. of Civil and Environmental Eng.

Sejong University

98 Kunja-dong, Kwangjin-gu

Seoul 143-747, South Korea

Tel.: 82-2-3408-3294

Fax: 82-2-3408-3332

Email: clee@sejong.ac.kr

April 13, 2007

\section{R. Cengiz Ertekin \\ Professor}

Dept. of Ocean and Resources Engineering

University of Hawaii, Honolulu, HI 96822, USA

Email: ertekin@hawaii.edu

Dear Professor Ertekin,

I submit our revised manuscript and replies to the reviewers' requests for the paper "Internal Generation of Waves: Delta Source Function Method and Source Term Addition Method" to be published in Ocean Engineering. I underlined all the changes in the revised manuscript in order to improve our manuscript following the reviewers' suggestions. Thank you very much.

Sincerely yours,

Changhoon Lee 


\section{Reply to referee's comments}

\section{Internal Generation of Waves: Delta Source Function Method and Source Term Addition Method by Kim/Lee/Suh, OE-D-06-00042}

We appreciate the referee's interest and criticisms on our manuscript entitled "Internal generation of waves: line source method and source function method." We hope that the revision we made could have reflected the referee's comments in the manuscript though the referees may not be fully satisfied with the revision.

Referee No. 1's specific comments:

(Question No. 1) Are both source methods based on the linear wave assumption? If yes, could the authors explain theoretically why the methods can generate nonlinear cnoidal waves almost perfectly?

(Answer No. 1) As the referee's comments, the source function methods are derived on the base of the linear wave assumptions. Thus, the energy velocity $C_{e}$ is obtained from the linear waves. However, nonlinear cnoidal waves can be generated properly using the source function given by $s=2 \eta^{I} C_{e} \delta\left(x-x_{s}\right) \cos \theta$, where $\eta^{I}$ is the incident water surface elevation of cnoidal waves which have peaked crest and flat trough.

(Question No. 2) On p.12, the authors stated that "It is interesting that Schaffer and Sorensen (2006) couldn't get the energy velocity for the equations of Madsen and Sorensen". This statement does not seem to serve any professional purposes in the paper.

(Answer No. 2) We revised the manuscript following the referee's suggestion.

(Question No. 3) On p.26, near the middle of the page, the authors write "There is no "2" in the right side of Eq. (67) while there is "2" in the right side of Eq. (56)". The writing does not seem to be very formal. Maybe "no 2" can be changed to "not a factor of $2 "$. 
(Answer No. 3) We revised the manuscript following the referee's suggestion. 


\section{Reply to referee's comments}

\section{Internal Generation of Waves: Delta Source Function Method and Source Term Addition Method by Kim/Lee/Suh, OE-D-06-00042}

We appreciate the referee's interest and criticisms on our manuscript entitled "Internal generation of waves: line source method and source function method." We hope that the revision we made could have reflected the referee's comments in the manuscript though the referees may not be fully satisfied with the revision.

Referee No. 2's specific comments:

(Question No. 1) The computational method applied is outlined. Nothing is said or illustrated in a figure as to how accurate the solutions may be. For example, were the cnoidal waves generated compared with their analytical shapes?

(Answer No. 1) We compared the numerical solutions against the analytical solution of the cnoidal waves at the last paragraph of p. 31 to the first paragraph of p. 32 in the manuscript as: "Fig. 5 shows the comparison of numerically generated water surface elevations and envelopes of cnoidal waves at a time of $t=30 T$ against the target one. In the figure, the horizontal distance is normalized by the wavelength obtained by the linearized dispersion relation. In the inner domain, good agreements are observed between the numerical solutions and the exact on es even for the higher wave amplitude. As the wave height increases, the wavelength increases due to the nonlinear dispersion. In the sponge layer domain, the wave envelopes decay down to almost zero values. This shows the capability of gen erating nonlinear waves using the delta source function. As the wave height increases, small oscillations are found more significantly both in the trough-level surface elevation and the envelope. However, these oscillations are not amplified even after a long time."

In the authors' viewpoint, a more detailed comparison between the simulated and the target waves seems not necessary. We may have a little bit different solutions depending on the use of the grid system, either staggered or un-staggered, and on the methods of discretization, either finite difference, fin ite element, or finite volume, etc. 
(Question No. 2) According to page 28, the linear waves in Figure 2 are for the deltasource method. In this figure the normalized amplitude of linear waves are compared with water depth. The amplitude ratio is equal to one or reasonably close to one for all the cases shown. It appears that all of the models reproduce linear waves that are the same. It appears than that the linear forms of all of the systems of equations examined must (in some sense) reduce to a sum of terms essentially the same plus additional terms that are negligible (but dependent on the particular model). Although the author's suggest that the Peregrine model be used for shallow water only, without additional details on the robustness of the numerical method, this may not be fair.

(Answer No. 2) When generating waves using the source function, the wave models are reduced to the Helmholtz equation of $\frac{\partial^{2} \eta}{\partial x^{2}}+l^{2} \eta=s$ where the $x$-directional wave number $l$ is determined by the dispersion relation of each model. The Peregrine's model can give proper solutions only for shallow water due to the limitation of the model's dispersion relation. And thus, as shown in Fig. 2 (a), the Peregrine's model couldn't give accurate wave amplitudes in the depth range of $k h>0.5 \pi$.

(Question No. 3) In Figures 3 and 4 the wave shapes are illustrated for two systems of equations; one system is for shallow water the other is for deep water. It appears that the waves generated are linear. Then they point out what appears to be an anomaly in the wave picture that is guessed to be attributed to evanescent modes. It would be useful if the authors elaborated on this point further.

(Answer No. 3) The anomaly of the wave envelopes in the vicinity of the wave generation line are observed for the extended Boussinesq equations which are reduced to 4 th order ord inary differential equation over a constant depth. As being seen in the appendix, these equations include the evanescent modes. In the author's viewpoint, the source term seems to generate evanescent waves as well as propagating waves. However, this assumption is not theoretically demonstrated. In order to avoid any ambiguities, we removed the wave envelopes in the Figure 3 and 4 of the revised manuscript. The surface elevations showed that waves are accurately generated.

(Question No. 4) In the last figure cnoidal waves are illustrated for the deep water system of equations. They start by recalling that the "line source method was developed based on the assumption of the linearity of the wave model." The reviewer supposed 
that this is only a peculiarity of the wave generated and not the equations solved in the domain. The nonlinear equations must be solved in that domain otherwise nonlinear waves could not be possible. Are the waves generated by forcing the numerical model actually cnoidal? In deed they have broadened troughs and sharpened crests as compared with more sinusoidal waves.

(Answer No. 4) As the referee's comments, the source function methods are derived on the base of the linear wave assumptions. Thus, the energy velocity $C_{e}$ is obtained from the linear waves. However, nonlinear cnoidal waves can be generated properly using the source function given by $s=2 \eta^{I} C_{e} \delta\left(x-x_{s}\right) \cos \theta$, where $\eta^{I}$ is the incident water surface elevation of cnoidal waves which have peaked crest and flat trough.

(Question No. 5) Finally, it is not clear that a comparison of two methods was actually done. It is also not clear how useful the conclusions actually are. What should an engineer do with the conclusions? Which model is recommended by the authors? Is it the implementation that is recommended?

(Answer No. 5) Following the referee's suggestion, we included the following sentence at the first paragraph of p. 33 in the revised manuscript as: "By the fractional step splitting method, the two wave generation methods are proved to be identical to each other. However, the delta source function method is recommended to be used rather than the source term addition method in terms of the mathematical completeness because the delta source function is included in the original wave equation and then the resulting equations are discretized numerically."

(Question No. 6) The mathematics associated with deriving source functions for each system of equations is certainly interesting from a mathematical point of view. Reducing the extended equations to linear form certainly allowed the effective use of Green's functions to come up with the particular solution associated with the source term. Fig. 2 shows that all of the systems reproduce linear waves that are dispersive. Only two of the systems were examined to study the wave elevations for linear waves. Only one system was used to investigate the "surface" elevations of cnoidal waves.

(Answer No. 6) The horizontally two-dimensional wave equations are categorized into two types, i.e., the mild-slope equations and the Boussinesq equations. In our 
manuscript, we showed the surface elevations of linear waves for only two types of the wave models, i.e., the extended mild-slope equations of Lee et al.'s (2003) and the extended Boussinesq equations of Nwogu (1993). The solutions of the other wave models will be almost the same as Lee et al.'s or Nwogu's model solutions. Thus, we omitted the other solutions. We showed the surface elevations of nonlinear cnoidal waves using Nwogu's extended Boussinesq equations.

(Question No. 7) Since the paper is stated to examine internal waves, why do the authors display surface elevations in Figures 3-5? There seems to be a mix up as to what is actually being displayed. Internal waves are waves in density stratified basins. Hence, are the surface elevations imprints of the internal waves as they appear on the surface?

(Answer No. 7) The technique of internal generation of wave is to generate surface waves inside the computational domain. We do not study the generation of internal waves between two layers. 


\section{Reply to referee's comments}

\section{Internal Generation of Waves: Delta Source Function Method and Source Term Addition Method by Kim/Lee/Suh, OE-D-06-00042}

We appreciate the referee's interest and criticisms on our manuscript entitled "Internal generation of waves: line source method and source function method." We hope that the revision we made could have reflected the referee's comments in the manuscript though the referees may not be fully satisfied with the revision.

Referee No. 3's specific comments:

(Question No. 1) The end of section 4.2 suggests that the apparent error in the vicinity of the source for the result from Nwogu's equation is caused by the effect of evanescent modes. Why would evanescent modes be generated over a flat bottom, or what indication do the authors have that they are present in the result? If they are present in this result, why not in others? I don't think this speculation has a place in the manuscript.

(Answer No. 1) The anomaly of the wave envelopes in the vicinity of the wave generation line are observed for the extended Boussinesq equations which are reduced to 4 th order ord inary differential equation over a constant depth. As being seen in the appendix, these equations include an evanescent mode. In the author's viewpoint, the source term seems to generate an evanescent wave as well as a propagating wave. However, this assumption is not theoretically demonstrated. In order to avoid the ambiguous description, in the revised manuscript, we removed the wave envelopes in the Figure 3 and 4 . The surface elevations show that waves are accurately generated.

(Question No. 2) In section 4.3, the cnoidal wave solution being used is indeed not a solution of Nwogu's equations. The adjustment taking place in the generated wave is thus likely to be a result both of the numerical approach being studied as well as the nonlinear adjustment of the wave as it propagates. The example is thus not terribly informative. Why not run the example using the Peregrine equations, which the cnoidal wave solution given is more closely related?

(Answer No. 2) As the referee's comments, the cnoidal wave solution is not a solution 
of Nwogu's equations. In the revised manuscript, we showed the results using Peregrine's equations. The results are similar to those obtained by Nwogu's equations. 


\title{
Internal Generation of Waves: Delta Source Function Method and Source Term Addition Method
}

\author{
Gunwoo Kimª, Changhoon Lee ${ }^{b^{*}}$, Kyung-Duck $\operatorname{Suh}^{\mathrm{c}}$
}

a School of Civil, Urban, and Geosystem Engineering, Seoul National University, San

56-1, Shinlim-dong, Gwanak-gu, Seoul 151-742, South Korea

${ }^{\mathrm{b}}$ Department of Civil and Environmental Engineering, Sejong University, 98 Kunja-dong,

Kwangjin-gu, Seoul 143-747, South Korea

c School of Civil, Urban, and Geosystem Engineering \& Engineering Research Institute,

Seoul National University, San 56-1, Shinlim-dong, Gwanak-gu, Seoul 151-742, South

Korea

* Corresponding author. F ax: +82-2-3408-3332.

E-mail addresses: maossi1@snu.ac.kr (G. Kim), clee@sejong.ac.kr (C. Lee), kdsuh@snu.ac.kr (K.-D. Suh). 


\begin{abstract}
In this study, we investigate two internal wave generation methods in numerical modeling of time-dependent equations for water wave propagation, i.e., delta source function method and source term addition method, the latter of which has been called the line source method in literatures. We derive delta source functions for the Boussinesq type equations and extended mild-slope equations. By applying the fractional step splitting method, we show that the delta source function method is equivalent to the source term addition method employing the energy velocity. This suggests that the energy velocity should be used rather than the phase velocity for the transport of incident wave energy in the source term addition method. Finally, the performance of the delta source function method is verified by accurately generating nonlinear cnoidal waves as well as linear waves for horizon tally one-dimensional cases.
\end{abstract}

Keywords: Numerical generation of wave; Internal generation of waves; Source term addition; Source function; Energy velocity approach; Boussinesq equations; Extended mild-slope equations; Numerical experiment

\title{
1. Introduction
}

The propagation of water waves from ocean to coastal area is a dynamic phenomenon which can give human beings happiness some times or disaster in other times. In general, the wave models which predict the transformation of water waves are horizontally twodimensional equations in which the amplitude of vertical variation is assumed to be 
exponential or polynomial. The wave equations which have been developed until now may be categorized into two types, i.e., the Bouss in esq equations and the mild-slope equations. The Boussinesq equations are able to predict the propagation of nonlinear, weakly dispersive waves with high accuracy especially in shallow water. The mild-slope equations may exactly predict the propagation of linear, dispersive waves from deep to shallow waters.

In order to predict the wave field in a nearshore region using wave equations, waves should be generated at the offshore boundary and propagate in to the model domain. Waves reflected from the model domain should pass through the offshore boundary without any numerical disturbances. Otherwise, the waves re-reflected at the offshore boundary may influence the numerical solution in the model domain. In order to avoid the re-reflection problem at the wave generation boundary, internal wave generation techniques have been used along with the sponge layers which are placed at the offshore boundary. The internal wave generation techniques are categorized into the source term addition method and the source function method, the former of which has been called the line source method in literatures. In the source term addition method, we add the water surface elevation of desired energy to the corresponding values that are computed by the model equations. In the source function method, we add an appropriate source function to the model equation.

The source term addition method was first used by Larsen and Dancy (1983) in the Boussinesq equations of Peregrine (1967) by using the phase velocity $C$ for the transport of water mass. Later, Lee and Suh (1998) found that the use of $C$ in the Peregrine's equations was successful because the equations were applicable only in shallow water and tests were made only in shallow water where the phase velocity $C$ is 
almost the same as the energy velocity $C_{e}$. The energy velocity is the group velocity derived from each model equation. They argued that the velocity of disturbances caused by the incident wave is the energy velocity. After testing the use of the two velocities of $C$ and $C_{e}$ in shallow to deep waters, Lee et al. (2001) found that the use of $C_{e}$ instead of $C$ yields a proper wave energy in the extended Boussineq equations of Nwogu (1993) which is applicable in deep water with a small error. Recently, Lee and Yoon (2007) developed a technique of internally generating multi-directional waves on an arc in a rectangular grid system using the source term addition method. The technique generated wave energy much closer to the target energy than the state-of-the-art technique (Lee and Suh, 1998) of generating waves on wave generation lines only.

A two-grid source function was first used by Madsen and Larsen (1987) in the mildslope equations. Later, Wei et al. (1999) used a Gaussian source function in the nonlinear shallow water equations and the Boussinesq equations of Peregrine (1967) and Nwogu (1993). In order to obtain an explicit relation between the target waves and the waves by the source function, they solved the linearized model equations including the source function using the Green function method. Recently, Bellotti et al. (2003) derived a delta source function for the elliptic mild-slope equation using the Fourier transform technique. More recently, Kim et al. (2006) obtained the Gaussian source functions for the extended mild-slope equations of Suh et al. (1997) and Lee et al. (2003b) using the Green function method.

A vertical source function has also been used by Brorsen and Larsen (1987) who included the source function in the Laplace equation of the velocity potential. The vertical source function was defined by the delta function. They used the source function of both linear and nonlinear waves in the boundary in tegral equation method. 
The source term addition method and the source function method had been developed separately, and their relationship had not been investigated yet. Recently, Schäffer and Sørensen (2006) made theoretical derivations to get the energy velocities for the transport of incident wave energy in the source term addition method. They added the delta source function to the mass conservation type equation, integrated asymptotically the resulting equation at the generation point, and thus they obtained an explicit relation of the source function to the energy velocity. However, they couldn't get the energy velocity for the extended Boussinesq equations of Madsen and Sørensen (1992) because the source function couldn't be explicitly related to the energy velocity.

In this study, firstly, we derive the delta source function and the energy velocities in the source term addition method for various types of wave equations available to date. To do this, we add the delta source function to the mass conservation type equation, and solve the linearized model equations including the source function by using the Green function method. Secondly, the fractional step splitting method is used to find the relationship between the source term addition method and the source function method. The relation explains why the energy velocity should be used instead of the phase velocity in the source term addition method. Thirdly, numerical experiments are conducted to accurately generate nonlinear cnoidal waves as well as linear waves using the delta source function method in a finite difference system. Finally, a brief summary is given. 


\section{Delta Source Function Method}

Wei et al. (1999) obtained a particular solution for the wave equations including a source function by applying the Green's function method. They selected the amplitude of the source function as a Gaussian function in order to overcome the discontinuity of the source at the wave generation line. Due to the smooth shape of the Gaussian function, waves can be generated without numerical disturbances regardless of numerical methods.

But at least several grids are needed to guarantee the spatial resolution of the source function. With the Gaussian source function, we cannot find the relation between the source term addition method and the source function method because the source term addition method needs just one grid point to generate waves. Therefore, in the present study, we select a delta function for the amplitude of the source functions $s$ as

$s(x, y, t)=\widetilde{s} \exp \{i(m y-\omega t)\}$

where $\omega$ is the angular frequency, $m(=k \sin \theta)$ is the $y$-directional wave number, $\theta$ is the direction of incident waves from the $x$-axis, and $i$ is the imaginary unit. In the above equation $\widetilde{s}$ is a source function without the $y$-directional and time harmonic terms which is defined as 
$\widetilde{s}=D \delta\left(x-x_{s}\right)$

In the above equation, $D$ is the amplitude of the source function, $\delta$ is the delta function, and $x_{s}$ is the location of the source point. In the following sub-sections, we obtain the source function $s$ for each wave model of the Boussinesq equations and the mild-slope equations by equating the particular solution with the target value.

\subsection{Source function for Boussinesq equations of Peregrine (1967)}

The Bouss inesq equations of Peregrine (1967) are given by

$$
\begin{aligned}
& \frac{\partial \eta}{\partial t}+\nabla \cdot\{(h+\eta) \overline{\mathbf{u}}\}=0 \\
& \frac{\partial \overline{\mathbf{u}}}{\partial t}+(\overline{\mathbf{u}} \cdot \nabla) \overline{\mathbf{u}}+g \nabla \eta-\frac{1}{2} h \nabla\left\{\nabla \cdot\left(h \frac{\partial \overline{\mathbf{u}}}{\partial t}\right)\right\}+\frac{1}{6} h^{2} \nabla\left(\nabla \cdot \frac{\partial \overline{\mathbf{u}}}{\partial t}\right)=0
\end{aligned}
$$

where $\eta$ is the surface elevation, $\overline{\mathbf{u}}$ is the depth-averaged horizontal velocity and $h$ is the still water depth, and $\nabla$ is the horizontal gradient operator. For sinusoidal waves over a flat bottom, a linearized form of Eq. (4) gives the relation between $\eta$ and $\bar{\phi}$ as

$$
\eta=i \frac{\omega}{g}\left\{1+\frac{1}{3}(k h)^{2}\right\} \bar{\phi}
$$


where the velocity potential $\bar{\phi}$ is given by the relation as

$$
\overline{\mathbf{u}}=\nabla \bar{\phi}
$$

We add the source function $s$ in the right side of Eq. (3). Then, we substitute Eq. (6) into the linearized forms of Eqs. (3) and (4) over a constant water depth. Further, we combine these equations in favor of $\bar{\phi}$. Finally, integrating the combined equation in space and using the relation of Eq. (5), we get the following equation

$$
\frac{\partial^{2} \eta}{\partial t^{2}}-g h \nabla^{2} \eta-\frac{h^{2}}{3} \nabla^{2} \frac{\partial^{2} \eta}{\partial t^{2}}=-i \omega\left\{1+\frac{(k h)^{2}}{3}\right\} s
$$

In order to obtain the relationship between the source function $s$ and the surface elevation $\eta$, a particular solution of the differential equation including the source function should be obtained. Let the surface elevation propagating from the source point of $x=x_{s}$ be defined as

$$
\eta=\tilde{\eta} \exp \{i(m y-\omega t)\}
$$

where $\tilde{\eta}$ is the variable without the $y$-directional and time harmonic terms of the surface elevation. Substituting Eqs. (1) and (8) into Eq. (7) yields the following one- 
dimensional Helmholtz equation

$$
\frac{d^{2} \widetilde{\eta}}{d x^{2}}+l^{2} \widetilde{\eta}=i \frac{k^{2}}{\omega}\left\{1+\frac{(k h)^{2}}{3}\right\} \widetilde{s}
$$

where $l(=k \cos \theta)$ is the $x$-directional wave number. Using the results in the Appendix A.1, we obtain the particular solution of Eq. (9) as

$$
\tilde{\eta}=\frac{k^{2}}{2 \omega l}\left\{1+\frac{(k h)^{2}}{3}\right\} D \exp \left\{ \pm i l\left(x-x_{s}\right)\right\}
$$

where the upper plus and lower minus signs, respectively, represent the right- and leftgoing waves from the source point of $x=x_{s}$. The amplitude of the source function $D$ can be determined by equating Eq. (10) with the target value of $\tilde{\eta}=a \exp \left\{ \pm i l\left(x-x_{s}\right)\right\}$. Thus, using Eqs. (1) and (2), we get the source function given by

$s=2 \eta^{I} C_{e} \delta\left(x-x_{s}\right) \cos \theta$

where $\cos \theta=l / k, C_{e}$ is the energy velocity for Peregrine's Boussinesq equations (Lee and Suh., 1998) given by 


$$
C_{e}=\frac{\omega}{k\left(1+\frac{(k h)^{2}}{3}\right)}
$$

and $\eta^{I}$ is the water surface elevation of incident waves given by

$$
\eta^{I}=a \exp \{i(m y-\omega t)\}
$$

\subsection{Source function for extended Boussinesq equations of Madsen and Sørensen (1992)}

The extended Boussinesq equations of Madsen and Sørensen (1992) are given by

$$
\begin{aligned}
& \frac{\partial \eta}{\partial t}+\frac{\partial P}{\partial x}+\frac{\partial Q}{\partial y}=0 \\
& \frac{\partial P}{\partial t}+\frac{\partial}{\partial x}\left(\frac{P^{2}}{d}\right)+\frac{\partial}{\partial y}\left(\frac{P Q}{d}\right)+g d \frac{\partial \eta}{\partial x}-\left(B+\frac{1}{3}\right) h^{2}\left(\frac{\partial^{3} P}{\partial x^{2} \partial t}+\frac{\partial^{3} Q}{\partial x \partial y \partial t}\right) \\
& -B g h^{3}\left(\frac{\partial^{3} \eta}{\partial x^{3}}+\frac{\partial^{3} \eta}{\partial x \partial y^{2}}\right)-h \frac{\partial h}{\partial x}\left(\frac{1}{3} \frac{\partial^{2} P}{\partial x \partial t}+\frac{1}{6} \frac{\partial^{2} Q}{\partial y \partial t}+2 B g h \frac{\partial^{2} \eta}{\partial x^{2}}+B g h \frac{\partial^{2} \eta}{\partial y^{2}}\right) \\
& -h \frac{\partial h}{\partial y}\left(\frac{1}{6} \frac{\partial^{2} Q}{\partial x \partial t}+B g h \frac{\partial^{2} \eta}{\partial x \partial y}\right)=0 \\
& \frac{\partial Q}{\partial t}+\frac{\partial}{\partial x}\left(\frac{P Q}{d}\right)+\frac{\partial}{\partial y}\left(\frac{Q^{2}}{d}\right)+g d \frac{\partial \eta}{\partial y}-\left(B+\frac{1}{3}\right) h^{2}\left(\frac{\partial^{3} Q}{\partial y^{2} \partial t}+\frac{\partial^{3} P}{\partial x \partial y \partial t}\right) \\
& -B g h^{3}\left(\frac{\partial^{3} \eta}{\partial y^{3}}+\frac{\partial^{3} \eta}{\partial x^{2} \partial y}\right)-h \frac{\partial h}{\partial y}\left(\frac{1}{3} \frac{\partial^{2} Q}{\partial y \partial t}+\frac{1}{6} \frac{\partial^{2} P}{\partial x \partial t}+2 B g h \frac{\partial^{2} \eta}{\partial y^{2}}+B g h \frac{\partial^{2} \eta}{\partial x^{2}}\right) \\
& -h \frac{\partial h}{\partial x}\left(\frac{1}{6} \frac{\partial^{2} P}{\partial y \partial t}+B g h \frac{\partial^{2} \eta}{\partial x \partial y}\right)=0
\end{aligned}
$$

where $(P, Q)$ is the depth-integrated velocity, $d=h+\eta$ is the total water depth, and the value of the parameter $B$ is chosen to optimize the dispersion characteristics. Adding the source function $s$ in the right side of Eq. (14), and combining linearized 
forms of Eqs. (14) - (16) in favor of $\eta$ for a flat bottom yield the following equation

$$
\frac{\partial^{2} \eta}{\partial t^{2}}-g h \nabla^{2} \eta-\left(B+\frac{1}{3}\right) h^{2} \nabla^{2} \frac{\partial^{2} \eta}{\partial t^{2}}+B g h^{3} \nabla^{2}\left(\nabla^{2} \eta\right)=\frac{\partial s}{\partial t}-\left(B+\frac{1}{3}\right) h^{2} \nabla^{2} \frac{\partial s}{\partial t}
$$

Substituting Eqs. (1) and (8) into the above equation yields a one-dimensional differential equation given by

$C_{1} \frac{d^{4} \widetilde{\eta}}{d x^{4}}+C_{2} \frac{d^{2} \widetilde{\eta}}{d x^{2}}+C_{3} \tilde{\eta}=i \omega\left\{\widetilde{s}-\left(B+\frac{1}{3}\right) h^{2}\left(\frac{d^{2} \widetilde{s}}{d x^{2}}-m^{2} \widetilde{s}\right)\right\}$

where

$$
\begin{aligned}
& C_{1}=-B g h^{3} \\
& C_{2}=g h-\left(B+\frac{1}{3}\right) \omega^{2} h^{2}+2 B g h^{3} m^{2} \\
& C_{3}=\omega^{2}-g h m^{2}+\left(B+\frac{1}{3}\right) \omega^{2} h^{2} m^{2}-B g h^{3} m^{4}
\end{aligned}
$$

Using the results in the Appendix A.2, we obtain the particular solution of Eq. (18) as

$$
\widetilde{\eta}=\frac{\omega k\left\{1+\left(B+\frac{1}{3}\right)(k h)^{2}\right\}}{2\left\{\omega^{2}+B g k^{4} h^{3}\right\}} \frac{D}{\cos \theta} \exp \left\{ \pm i l\left(x-x_{s}\right)\right\}
$$

The amplitude of the source function $D$ can be determined by equating Eq. (20) with the target value of $\tilde{\eta}=a \exp \left\{ \pm i l\left(x-x_{s}\right)\right\}$. Thus, using Eqs. (1) and (2), we get the source function given by Eq. (11) where $\eta^{I}$ is given in Eq. (13) and $C_{e}$ is the energy velocity for the extended Boussinesq equations of Madsen and Sørensen given by 


$$
C_{e}=\frac{\omega}{k}\left[1-\frac{(k h)^{2}}{3\left\{1+B(k h)^{2}\right\}\left\{1+\left(B+\frac{1}{3}\right)(k h)^{2}\right\}}\right]
$$

Schäffer and Sørensen (2006) added the delta source function to the mass conservation type equation of Madsen and Sørensen, integrated asymptotically the resulting equation at the generation point, and thus obtained an explicit relation of the source function to the energy velocity. However, for the equations of Madsen and Sørensen, the source function cannot be explicitly related to the energy velocity because the spatial derivative of the source function appears in addition to the function itself as in Eq. (17).

\subsection{Source function for extended Boussinesq equations of Nwogu (1993)}

The extended Boussinesq equations of Nwogu (1993) are given by

$$
\begin{aligned}
& \frac{\partial \eta}{\partial t}+\nabla \cdot\left\{(h+\eta) \mathbf{u}_{\alpha}\right\}+\nabla \cdot\left[\left(\frac{z_{\alpha}^{2}}{2}-\frac{h^{2}}{6}\right) h \nabla\left(\nabla \cdot \mathbf{u}_{\alpha}\right)+\left(z_{\alpha}+\frac{h}{2}\right) h \nabla\left\{\nabla \cdot\left(h \mathbf{u}_{\alpha}\right)\right\}\right]=0 \\
& \frac{\partial \mathbf{u}_{\alpha}}{\partial t}+\left(\mathbf{u}_{\alpha} \cdot \nabla\right) \mathbf{u}_{\alpha}+g \nabla \eta+z_{\alpha}\left[\frac{z_{\alpha}}{2} \nabla\left(\nabla \cdot \frac{\partial \mathbf{u}_{\alpha}}{\partial t}\right)+\nabla\left\{\nabla \cdot\left(h \frac{\partial \mathbf{u}_{\alpha}}{\partial t}\right)\right\}\right]=0
\end{aligned}
$$

where $\mathbf{u}_{\alpha}$ is the horizontal velocity at a certain elevation of $z=z_{\alpha}$. For sinusoidal waves over a flat bottom, a linearized form of Eq. (23) gives the relation between $\eta$ and $\phi_{\alpha}$ as 
$\eta=i \frac{\omega}{g}\left\{1-\alpha(k h)^{2}\right\} \phi_{\alpha}$

where $\alpha=\left(z_{\alpha} / h\right)^{2} / 2+z_{\alpha} / h$ and the velocity potential $\phi_{\alpha}$ is given by the relation as

$\mathbf{u}_{\alpha}=\nabla \phi_{\alpha}$

We add the source function $s$ in the right side of Eq. (22). Then, we substitute Eq. (25) into the linearized forms of Eqs. (22) and (23) over a constant water depth. Further, we combine these equations in favor of $\phi_{\alpha}$. Finally, integrating the combined equation in space and using the relation of Eq. (24), we get the following equation

$\frac{\partial^{2} \eta}{\partial t^{2}}-g h \nabla^{2} \eta-\left(\alpha+\frac{1}{3}\right) g h^{3} \nabla^{2}\left(\nabla^{2} \eta\right)+\alpha h^{2} \nabla^{2} \frac{\partial^{2} \eta}{\partial t^{2}}=-i \omega\left\{1-\alpha(k h)^{2}\right\}_{s}$

Substituting Eqs. (1) and (8) into the above equation yields a one-dimensional differential equation given by

$C_{1} \frac{d^{4} \tilde{\eta}}{d x^{4}}+C_{2} \frac{d^{2} \tilde{\eta}}{d x^{2}}+C_{3} \tilde{\eta}=C_{4} \widetilde{s}$

where

$$
\begin{aligned}
& C_{1}=\left(\alpha+\frac{1}{3}\right) g h^{3} \\
& C_{2}=g h+\alpha \omega^{2} h^{2}-2\left(\alpha+\frac{1}{3}\right) g h^{3} m^{2} \\
& C_{3}=\omega^{2}-g h m^{2}-\alpha \omega^{2} h^{2} m^{2}+\left(\alpha+\frac{1}{3}\right) g h^{3} m^{4}
\end{aligned}
$$




$$
C_{4}=i \omega\left\{1-\alpha(k h)^{2}\right\}
$$

Using the results in the Appendix A.3, we obtain the particular solution of Eq. (27) as

$$
\tilde{\eta}=\frac{\omega k\left\{1-\alpha(k h)^{2}\right\}}{2\left\{\omega^{2}-\left(\alpha+\frac{1}{3}\right) g k^{4} h^{3}\right\}} \frac{D}{\cos \theta} \exp \left\{ \pm i\left(x-x_{s}\right)\right\}
$$

The amplitude of the source function $D$ can be determined by equating Eq. (29) with the target value of $\tilde{\eta}=a \exp \left\{ \pm i l\left(x-x_{s}\right)\right\}$. Thus, using Eqs. (1) and (2), we get the source function given by Eq. (11) where $\eta^{I}$ is given in Eq. (13) and $C_{e}$ is the energy velocity for the extended Boussinesq equations of Nwogu (Lee et al., 2001) given by

$$
C_{e}=\frac{\omega}{k}\left[1-\frac{(k h)^{2}}{\left.3\left\{1-\alpha(k h)^{2}\right\} 1-\left(\alpha+\frac{1}{3}\right)(k h)^{2}\right\}}\right]
$$

\subsection{Source function for elliptic extended mild-slope equation of Massel (1993)}

The elliptic extended mild-slope equation of Massel (1993) is given by

$$
\nabla \cdot\left(C C_{g} \nabla \hat{\varphi}\right)+\left\{k^{2} C C_{g}-\omega^{2} R_{1}(\nabla h)^{2}-\omega^{2} R_{2} \nabla^{2} h\right\} \hat{\varphi}=0
$$

where $\hat{\varphi}=\varphi \exp (i \omega t)$ and $\varphi$ is the velocity potential at the mean water level, $C$ and $C_{g}$ are the phase and group velocities, respectively, and $R_{1}$ and $R_{2}$ are the 
coefficients related to the second order bottom effect. For a flat bottom, adding the source function $s$ in the right side of Eq. (31), defining the variable $\hat{\eta}=\eta \exp (i \omega t)$ and using the relation of $\hat{\eta}=-i(\omega / g) \hat{\varphi}$ give the following equation

$\nabla^{2} \hat{\eta}+k^{2} \hat{\eta}=i \frac{\omega}{g C C_{g}} s$

Substituting $\hat{\eta}=\tilde{\eta} \exp ($ imy $)$ and $s=\widetilde{s} \exp ($ imy $)$ into Eq. (32) yields a one-dimensional Helmholtz equation given by

$$
\frac{d^{2} \tilde{\eta}}{d x^{2}}+l^{2} \widetilde{\eta}=i \frac{\omega}{g} \frac{\widetilde{s}}{C C_{g}}
$$

Using the results in the Appendix A.4, we obtain the particular solution of Eq. (33) as

$$
\widetilde{\eta}(x)=\frac{1}{2 g C_{g} \cos \theta} D \exp \left\{ \pm i l\left(x-x_{s}\right)\right\}
$$

The amplitude of the source function $D$ can be determined by equating Eq. (34) with the target value of $\tilde{\eta}=a \exp \left\{ \pm i l\left(x-x_{s}\right)\right\}$. Thus, using the relation of $s=\tilde{s} \exp ($ imy $)$ and Eq. (2), we get the source function given by

$$
s=2 \eta^{I} \exp (i \omega t) g C_{e} \delta\left(x-x_{s}\right) \cos \theta
$$

where $\eta^{I}$ is given in Eq. (13) and $C_{e}=C_{g}$ is the energy velocity for the extended 
mild-slope equation of Massel. When the direction of the incident wave is perpendicular to the $y$-axis, i.e., $\theta=0$, Eq. (35) is the same as the source function derived by Bellotti et al. (2003).

\subsection{Source function for extended mild-slope equations of Suh et al. (1997)}

The extended mild-slope equations of Suh et al. (1997) are given by

$$
\begin{aligned}
& \frac{\partial \eta}{\partial t}+\nabla \cdot\left(\frac{\bar{C} \bar{C}_{g}}{g} \nabla \varphi\right)-\frac{\bar{\omega}^{2}-\bar{k}^{2} \bar{C} \bar{C}_{g}}{g} \varphi-\frac{\bar{\omega}^{2}}{g}\left\{\bar{R}_{1}(\nabla h)^{2}+\bar{R}_{2} \nabla^{2} h\right\} \varphi=0 \\
& \frac{\partial \varphi}{\partial t}+g \eta=0
\end{aligned}
$$

where $\varphi$ is the velocity potential at the mean water level and the over bar indicates the variables associated with the carrier angular frequency $\bar{\omega}$. Adding the source function $s$ in the right side of Eq. (36), combining Eqs. (36) and (37) in favor of $\varphi$ for a flat botto $\mathrm{m}$, and using the relation $\eta=-i(\omega / g) \varphi$ give the following equation

$$
\frac{\partial^{2} \eta}{\partial t^{2}}-\bar{C} \bar{C}_{g} \nabla^{2} \eta+\left(\bar{\omega}^{2}-\bar{k}^{2} \bar{C} \bar{C}_{g}\right) \eta=-i \omega s
$$

Substituting Eqs. (1) and (8) into the above equation yields a one-dimensional Helmholtz equation given by

$$
\frac{d^{2} \widetilde{\eta}}{d x^{2}}+l^{2} \widetilde{\eta}=i \frac{\omega}{\bar{C} \bar{C}_{g}} \widetilde{s}
$$


Using the results in the Appendix A.4, we obtain the particular solution of Eq. (39) as

$$
\widetilde{\eta}=\frac{\omega}{2 l \bar{C} \bar{C}_{g}} D \exp \left\{ \pm i l\left(x-x_{s}\right)\right\}
$$

The amplitude of the source function $D$ can be determined by equating Eq. (40) with the target value of $\tilde{\eta}=a \exp \left\{ \pm i l\left(x-x_{s}\right)\right\}$. Thus, using Eqs. (1) and (2), we get the source function given by Eq. (11) where $\cos \theta=l / k, \eta^{I}$ is given in Eq. (13) and $C_{e}$ is the energy velocity for the extended mild-slope equation of Suh et al. (Lee and Suh, 1998) given by

$$
C_{e}=\frac{\bar{C} \bar{C}_{g}}{C}
$$

\subsection{Source function for extended mild-slope equations of Lee et al. (1998)}

The extended mild-slope equations of Lee et al. (1998) are given by

$$
\begin{aligned}
& \frac{\partial \eta}{\partial t}+\frac{\nabla \cdot \mathbf{Q}}{\frac{\bar{C}_{g}}{\bar{C}}-\bar{R}_{1}(\nabla h)^{2}-\bar{R}_{2} \nabla^{2} h}=0 \\
& \frac{\partial \mathbf{Q}}{\partial t}+\bar{C} \bar{C}_{g} \nabla \eta=0
\end{aligned}
$$

where the flux $\mathbf{Q}$ is defined as $\mathbf{Q}=\overline{\mathbf{C}}_{\mathrm{g}} \eta$. Adding the source function $s$ in the right side of Eq. (42), and combining Eqs. (42) and (43) in favor of $\eta$ for a flat bottom give the following equation 
$\frac{\partial^{2} \eta}{\partial t^{2}}-\bar{C}^{2} \nabla^{2} \eta=-i \omega s$

Substituting Eqs. (1) and (8) into the above equation yields a one-dimensional Helmholtz equation given by

$$
\frac{d^{2} \widetilde{\eta}}{d x^{2}}+l^{2} \widetilde{\eta}=i \frac{k}{\bar{C}} \widetilde{s}
$$

Using the results in the Appendix A.4, we obtain the particular solution of Eq. (45) as

$$
\widetilde{\eta}=\frac{k}{2 l \bar{C}} D \exp \left\{ \pm i l\left(x-x_{s}\right)\right\}
$$

The amplitude of the source function $D$ can be determined by equating Eq. (46) with the target value of $\tilde{\eta}=a \exp \left\{ \pm i l\left(x-x_{s}\right)\right\}$. Thus, using Eqs. (1) and (2), we get the source function given by Eq. (11) where $\cos \theta=l / k, \eta^{I}$ is given in Eq. (13) and $C_{e}$ is the energy velocity for the extended mild-slope equation of Lee et al. (Lee and Suh, 1998) given by

$$
C_{e}=\bar{C}
$$




\subsection{Source function for extended mild-slope equation of Lee et al. (2003b)}

The extended mild-slope equation of Lee et al. (2003b) is given by

$$
\begin{aligned}
& \nabla \cdot\left(\bar{C} \bar{C}_{g} \nabla \varsigma\right)+\left\{\bar{k}^{2} \bar{C} \bar{C}_{g}+g \bar{u}_{1} \nabla^{2} h+g \bar{u}_{2}(\nabla h)^{2}\right\} \varsigma+i \nabla \cdot\left\{\frac{\partial\left(C C_{g}\right)}{\partial \omega} \nabla \frac{\partial \varsigma}{\partial t}\right\} \\
& +i\left\{\frac{\partial\left(k^{2} C C_{g}\right)}{\partial \omega}+g \frac{\overline{\partial u_{1}}}{\partial \omega} \nabla^{2} h+g \frac{\overline{\partial u_{2}}}{\partial \omega}(\nabla h)^{2}\right\} \frac{\partial \varsigma}{\partial t}=0
\end{aligned}
$$

where $\varsigma$ is related to $\eta$ by $\eta=\varsigma \exp (-i \bar{\omega} t) . \bar{u}_{1}$ and $\bar{u}_{2}$ are the coefficients mathematically equivalent to $-\bar{R}_{2} \varpi^{2} / g$ and $-\bar{R}_{1} \varpi^{2} / g$, respectively. For a flat bottom, adding the source function $s$ in the right side of Eq. (48) yields the following equation

$$
\bar{C} \bar{C}_{g} \nabla^{2} \varsigma+\bar{k}^{2} \bar{C} \bar{C}_{g} \varsigma+i \frac{\overline{\partial\left(C C_{g}\right)}}{\partial \omega} \nabla^{2} \frac{\partial \varsigma}{\partial t}+i \frac{\overline{\partial\left(k^{2} C C_{g}\right)}}{\partial \omega} \frac{\partial \varsigma}{\partial t}=s
$$

Substituting $\varsigma=\widetilde{\eta} \exp \{i(m y-\Delta \omega t)\}$ and $s=\widetilde{s} \exp \{i(m y-\Delta \omega t)\}$, where $\Delta \omega=\omega-\bar{\omega}$, into the above equation yields a one-dimensional Helmholtz equation given by

$$
\frac{d^{2} \widetilde{\eta}}{d x^{2}}+l^{2} \widetilde{\eta}=\frac{\widetilde{s}}{\bar{C} \bar{C}_{g}+\Delta \omega \frac{\overline{\partial\left(C C_{g}\right)}}{\partial \omega}}
$$

Using the results in the Appendix A.4, we obtain the particular solution of Eq. (50) as 


$$
\tilde{\eta}=\frac{-i}{2 l\left\{\bar{C} \bar{C}_{g}+\Delta \omega \frac{\partial\left(C C_{g}\right)}{\partial \omega}\right\}} D \exp \left\{ \pm i l\left(x-x_{s}\right)\right\}
$$

The amplitude of the source function $D$ can be determined by equating Eq. (51) with the target value of $\widetilde{\eta}=a \exp \left\{ \pm i l\left(x-x_{s}\right)\right\}$. Thus, using Eqs. (1) and (2), we get the source function given by

$s=i \varsigma^{I} C_{e}\left\{\frac{\overline{\partial\left(k^{2} C C_{g}\right)}}{\partial \omega}-k^{2} \frac{\overline{\partial\left(C C_{g}\right)}}{\partial \omega}\right\} \delta\left(x-x_{s}\right) \cos \theta$

where $\cos \theta=l / k, C_{e}$ is the energy velocity for the extended mild-slope equations of Lee et al. (2003b) given by

$$
C_{e}=2 k \frac{\bar{C} \bar{C}_{g}+\Delta \omega \frac{\overline{\partial\left(C C_{g}\right)}}{\overline{\partial\left(k^{2} C C_{g}\right)}}-k^{2} \frac{\partial \omega}{\partial \omega}}{\partial \omega}
$$

and $\varsigma^{I}$ is the surface elevation function of incident waves given by

$$
\varsigma^{I}=a \exp \{i(m y-\Delta \omega t)\}
$$




\section{Relation between Source Term Addition Method and Delta Source Function Method}

We first follow the previous studies to get the energy velocities for the time-dependent wave equations which are needed in the source term addition method. And then, we find the equivalence between the two line source methods, i.e., the source term addition method and the delta source function method for the time-dependent wave equations by using the fractional step splitting method.

\subsection{Source term addition method}

In the source term addition method, we add at each time step the water surface elevation $\eta^{*}$ of desired energy to the corresponding value $\eta^{\text {model }}$ that is computed by the time-dependent model equations. Thus, we have the following equation

$\eta^{n+1}=\eta^{\text {model }}+\eta^{*}$

where the superscript $n+1$ denotes the time step in which the surface elevation is predicted. When the wave generation line is parallel to the $y$-axis, the value $\eta^{*}$ which is added at the wave generation line is given by (Lee and Suh, 1998)

$\eta^{*}=2 \eta^{I} \frac{C_{e} \Delta t}{\Delta x} \cos \theta$

where $\eta^{I}$ is the water surface elevation of incident waves, $C_{e}$ is the energy velocity, 
$\theta$ is the direction of incident waves from the $x$-axis, $\Delta x$ is the grid size in the $x$-axis, and $\Delta t$ is the time step. It is noted that only the $x$-directional component of the wave energy is transported, which is normal to the wave generation line. It is also noted that there is ' 2 ' in Eq. (56) because wave energy is transported in both directions from the wave generation line.

The energy velocity $C_{e}$ can be found by applying the geometric optics approach to each wave model for a flat bottom. Lee and Suh (1998) and Lee et al. (2001) derived in detail the energy velocity as well as the phase velocity for several wave models. The surface elevation $\eta$ can be defined as

$\eta=a \exp (i \Psi)$

where the amplitude $a$ modulates in horizontal space and time, $i$ is the imaginary unit, and the phase function $\Psi$ has the following relations with the angular frequency $\omega$ and the wave number components $(l, m)$ :

$$
\frac{\partial \Psi}{\partial t}=-\omega, \quad \nabla \Psi=( \pm l, m)
$$

where the upper plus and lower minus signs, respectively, represent the right- and left- 
going waves from the source line. Substituting Eq. (57) into the linearized governing equations and rearranging the imaginary part yield the energy velocities given by Eqs. (12), (21), (30), (41), and (47) for the wave equations of Peregrine (1967), Madsen and Sørensen (1992), Nwogu (1993), Suh et al. (1997), and Lee et al. (1998), respectively. For the extended mild-slope equation of Lee et al. (2003b), using the relations of $\varsigma=a \exp \{i(\Psi+\bar{\omega} t)\}$ and $\partial \varsigma / \partial t=-i \varsigma \Delta \omega$, where $\Delta \omega=\omega-\bar{\omega}$, and rearranging the imaginary part of the resulting equation yield the energy velocity given by Eq. (53).

\subsection{Fractional step splitting method}

In this section, the fractional step splitting method (LeVeque, 1998) is used to find the equivalence between the source term addition method and the delta source function method for the wave equations. We also show that the energy velocity should be used in the source term addition method.

If the source function method is used for the wave equations, the resulting equation would be a form of the conservation equation for $q$ including a source function $S$ :

$$
\frac{\partial q}{\partial t}+f(q)=S
$$

where $q$ is a physical variable and $f(q)$ is a function of $q$ which may have a spatial derivative of $q$ to consider the convection of the variable. The fractional step splitting method allows to split Eq. (59) into the homogeneous conservation equation

$$
\frac{\partial q}{\partial t}+f(q)=0
$$


and the ordinary differential equation

$\frac{d q}{d t}=S$

at each time step. Also, Eq. (61) can be discretized in time using a forward difference scheme as

$q^{n+1}=q^{\text {model }}+q^{*}$

where $q^{\text {model }}$ is obtained by solving the conservation Eq. (60) which does not include the source function and $q^{*}$ is obtained as

$q^{*}=S \Delta t$

The mathematical form of Eq. (55) in the source term addition method is equal to Eq. (62) in the delta source function method. If the physical variable $q$ is the water surface elevation $\eta$, the added value $q^{*}$ in Eq. (63) is the added value $\eta^{*}$ in Eq. (56). In a rectangular grid system, the delta function may be represented as a unit impulse function given by

$$
\delta\left(x-x_{s}\right)= \begin{cases}\frac{1}{\Delta x}, & \left|x-x_{s}\right| \leq \frac{\Delta x}{2} \\ 0, & \left|x-x_{s}\right|>\frac{\Delta x}{2}\end{cases}
$$

The mass conservation type Eqs. (3), (14), (22), (32), and (42) in the wave equations of 
Peregrine (1967), Madsen and Sørensen (1992), Nwogu (1993), Suh et al. (1997), and Lee et al. (1998), respectively, have the same forms as the homogeneous conservation Eq. (60) in terms of $\eta$. Adding the source functions given by Eq. (11) to the right sides of the correspond ing mass conservation equations and solving them by the fractional step splitting method give $\eta^{*}$ for each equation. Using Eq. (64) for $\delta\left(x-x_{s}\right)$ in the obtained $\eta^{*}$ and comparing the resulting $\eta^{*}$ with Eq. (56), we obtain the energy velocity $C_{e}$ which is obtained by the geometrical optics approach and used in the source term addition method.

For example, the mass conservation equation of Peregrine (1967) including the source function, Eq. (11), is given by

$$
\frac{\partial \eta}{\partial t}+\nabla \cdot\{(h+\eta) \overline{\mathbf{u}}\}=2 \eta^{I} C_{e} \delta\left(x-x_{s}\right) \cos \theta
$$

Solving this equation by the fractional step splitting method gives the added value $\eta^{*}$. Using Eq. (64) for $\delta\left(x-x_{s}\right)$ in the obtained $\eta^{*}$ and comparing the resulting $\eta^{*}$ with Eq. (56) yield the energy velocity given by Eq. (12) which was used in the source term addition method.

Lee et al.'s (2003b) extended mild-slope Eq. (48), which includes the source function, is not a form of the conservation Eq. (59). By using the relation of $\nabla^{2} \varsigma=-k^{2} \varsigma$, we can modify Eq. (49) to be a form of the conservation equation in terms of $\varsigma$ as 


$$
\frac{\partial \varsigma}{\partial t}-i \frac{\left(\bar{k}^{2}-k^{2}\right) \bar{C} \bar{C}_{g}}{\frac{\partial\left(k^{2} C C_{g}\right)}{\partial \omega}-k^{2} \frac{\overline{\partial\left(C C_{g}\right)}}{\partial \omega}} \varsigma=-i \frac{s}{\frac{\partial\left(k^{2} C C_{g}\right)}{\partial \omega}-k^{2} \frac{\overline{\partial\left(C C_{g}\right)}}{\partial \omega}}
$$

Therefore, the added value $\varsigma^{*}$ at each time step is given by

$$
\begin{aligned}
\varsigma^{*} & =-i \frac{s}{\frac{\partial\left(k^{2} C C_{g}\right)}{\partial \omega}-k^{2} \frac{\overline{\partial\left(C C_{g}\right)}}{\partial \omega}} \Delta t \\
& =\varsigma^{I} \frac{C_{e} \Delta t}{\Delta x} \cos \theta
\end{aligned}
$$

In order to obtain the energy velocity, we used the source function given by Eq. (52) as well as the delta function given by Eq. (64). There is not a factor of 2 in the right side of Eq. (67) while there is a factor of 2 in the right side of Eq. (56) which is applied to all other wave models.

Schäffer and Sørensen (2006) also derived the source functions given in Eq. (11) for the extended Boussinesq equations of Nwogu (1993) and the mild-slope equations of Radder and Dingemans (1985) and Copeland (1985). They considered for only horizontally one-dimensional cases, and thus did not explain the presence of $\cos \theta$ in Eq. (11) for horizontally two-dimensional cases. Also, they did not show that, from the delta source function method, the added value of $\eta^{*}$ in the source term addition method should be related to the en ergy velocity $C_{e}$ as given in Eq. (56). However, it is hard to get the relation of Eq. (56) directly from the delta source function method. Furthermore, without the fractional step splitting method which was used in the present study, the added value of $\varsigma^{*}$ for Lee et al.'s (2003b) cannot be related to the energy velocity $C_{e}$ 
as given in Eq. (67).

It should be noted that the source function may have a different dimension depending on the wave equation to which the source function is added. The source function has the dimension of $\left[L T^{-1}\right]$ for the equations of Peregrine (1967), Madsen and Sørensen (1992), Nwogu (1993), Suh et al. (1997), and Lee et al. (1998). And, the source function

has the dimension of $\left[L^{2} T^{-3}\right]$ and $\left[L T^{-2}\right]$ for the equations of Massel (1993) and Lee et al. (2003b), respectively. Here, $L$ and $T$ denote the length and time, respectively.

\section{Numerical Experiments}

By numerical experiments, Lee and Suh (1998) verified the source term addition method with the use of the energy velocity in the mild-slope equations of Radder and Dingemans (1985) and Copeland (1985). They also proved its applicability in generating multi-directional random waves using three wave generation lines which surround the concerned region. Lee et al. (2001) verified the source term addition method with the use of the energy velocity in the extended Boussin esq equations of Nwogu (1993). They also generated nonlinear cnoidal waves in shallow water.

In this section, we generate linear waves using the delta source functions in all the types of the Boussinesq type equations and the extended mild-slope equations. We also generate cnoidal waves using the extended Boussinesq equations of Peregrine (1967). 


\subsection{Finite difference method}

To minimize wave reflection from the boundaries, sponge layers are placed at the outside boundaries by dissipating wave energy inside the sponge layers. Thus, the damping coefficient $D_{s}$ is given by

$D_{s}= \begin{cases}\frac{\exp (d / W)-1}{\exp (1)-1}, & \text { inside sponge layer } \\ 0, & \text { outside sponge layer }\end{cases}$

where $d$ is the distance from the starting point of the sponge layer and $W$ is the thickness of the sponge layer.

All the Boussinesq type models are descretized in time by the composite $4^{\text {rd }}$ order Adams-Bashforth-Moulton predictor-corrector scheme of Wei and Kirby (1995). . A 9point filtering technique (Shapiro, 1970) was used to smooth out numerical noises in the solution of the Boussinesq equations. The extended mild-slope equations of Suh et al. (1997) are discretized in time by the $4^{\text {th }}$ order Adams-Moulton predictor-corrector scheme (Kirby et al., 1992). The extended mild-slope equations of Lee et al. (1998) are discretized by a leap-frog method in a staggered grid in time and space. The extended mild-slope equations of Lee et al. (2003b) are discretized in time and space by the CrankNicols on scheme.

\subsection{Generation of linear waves}

Using the delta source function method we generate linear waves for all the Boussinesq type and mild-slope type equations. The computational domain consists of an inner domain of $15 L$, where $L$ is the wavelength, and two sponge layers with the 
thickness of $2.5 \mathrm{~L}$ at the outside boundaries. The source point is located at the center of the inner domain (see Fig. 1). The water depth is $1 \mathrm{~m}$ and the amplitude of target water surface elevation is $0.001 \mathrm{~m}$. The wave amplitudes are measured one wavelength apart from the wave generation line. These are normalized by the target amplitude.

Figs. 2 (a) - (f) show the normalized wave amplitudes versus relative water depth for the Boussinesq type models of Peregrine (1967), Madsen and Sørensen (1992), and Nwogu (1993), and the extended mild-slope equations of Suh et al. (1997), Lee et al. (1998), and Lee et al. (2003b). On the whole, the amplitudes of the generated waves are almost equal to the target amplitude. Although the wave energy can be accurately generated even in a deep water for the extended Boussinsq equations of Madsen and Sørensen (1992) and Nwogu (1993), use of these models should be limited up to the intermediate water depth to guarantee a good dispersion characteristics (Lee et al., 2003a).

For Peregrine's Boussinesq equations, the wave amplitudes are somewhat different from the target one at water depth of $k_{e} h>0.7 \pi$ where $k_{e}$ is the wave number obtained by the dispersion relation for linear Stokes waves. This is because the conventional Boussinesq equations cannot predict wave amplitudes as well as wavelengths due to the limitation in the dispersion relation when the water depth becomes large. Thus, in the case of deep water with $k_{e} h=\pi$, waves could not be generated. The dispersion relation for the Peregrine's Boussinesq equations is

$$
C=\frac{\omega}{k}=\sqrt{\frac{g h}{1+\frac{(k h)^{2}}{3}}}
$$


From Eq. (69), the wave number $k$ may be determined as

$$
k=\sqrt{\frac{\omega^{2}}{g h-\frac{\omega^{2} h^{2}}{3}}}
$$

The denominator in the above equation should be greater than zero to get a real value of $k$. Using the linear dispersion relation of $\omega^{2}=g k_{e} \tanh k_{e} h$, we can see that the relative water depth $k_{e} h$ should satisfy the condition of $k_{e} h<0.96 \pi$. However, the model should be used only in shallow water to guarantee a good dispersion relation.

Figs. 3 and 4 show the surface elevations generated for the extended mild-slope equation of Lee et al. (2003b) and the extended Boussinesq equations of Nwogu (1993), respectively, in a shallow water $\left(k_{e} h=0.1 \pi\right)$ and a deep water $\left(k_{e} h=\pi\right)$. For both the extended mild-slope equation and extended Boussinesq equations, the normalized amplitudes are almost equal to one in the inner domain. Wave amplitudes decay down to almost zero values in the sponge layer domain.

\subsection{Generation of cnoidal waves}

The source term addition method was developed based on the assumption of the linearity of the wave model. However, using the source term addition method, Larsen and Dancy (1983) and Lee et al. (2001) generated cnoidal waves using the Boussinesq type equations of Peregrine (1967) and Nwogu (1993), respectively. Even though the linear energy velocity was used at one grid point in generating non linear waves, the wave models reproduced the propagation of nonlinear cnoidal waves. In this section, we generate cnoidal waves using the delta source function method in the Boussinesq 
The water surface elevation of cnoidal waves $\eta$ is given by

$\eta=\eta_{t}+H \mathrm{cn}^{2}\left(\frac{2 K t}{T} \mid m\right)$

where $H$ is the wave height, and $\eta_{t}$ is the elevation of wave trough given by

$\eta_{t}=\frac{H}{m}\left(1-m-\frac{E}{K}\right)$

where $\mathrm{cn}$ is the Jacobian elliptic function, $K$ is the complete elliptic integral of the first kind, $E$ is the complete elliptic integral of the second kind, and $m$ is the modulus which determines the wave shape. It should be noted that these cnoidal wave solutions are not necessarily the solutions to all sets of the Boussinesq equations and anomalies may show up in the computations unless the relative water depth is relatively small.

The computational domain is the same as the case of linear waves. The wave period is $T=20 \mathrm{~s}$ and the water depth is $h=10 \mathrm{~m}$, and thus the water depth is relatively shallow with $k_{e} h=0.1 \pi$. The wave heights are $H=1 \mathrm{~m}, 2 \mathrm{~m}$, and $3 \mathrm{~m}$, so the Ursell numbers are $U_{r}=(a / h) /\left(k_{e} h\right)^{2}=0.48,0.96$, and 1.44 , respectively, and the moduli are $m=$ $0.9360,0.9938$, and 0.9992 , respectively

Fig. 5 shows the comparison of numerically generated water surface elevations and envelopes of cnoidal waves at a time of $t=30 T$ against the target one. In the figure, the horizontal distance is normalized by the wavelength obtained by the linearized dispersion 
relation. In the inner domain, good agreements are observed between the numerical solutions and the exact ones even for the higher wave amplitude. As the wave height increases, the wavelength increases due to the nonlinear dispersion. In the sponge layer domain, the wave envelopes decay down to almost zero values. This shows the capability of generating nonlinear waves using the delta source function. As the wave height increases, small oscillations are found more significantly both in the trough-level surface elevation and the envelope. However, these oscillations are not amplified even after a long time.

\section{Conclusions}

In this study, we investigated two internal wave generation methods, i.e., delta source function method and source term addition method. The internal wave generation techniques have been used along with the sponge layers which are placed at the offshore boundary in order to avoid the re-reflection problem at the wave generation boundary. We derived delta source functions for the Boussinesq equations of Peregrine (1967), the extended Boussinesq equations of Madsen and Sørensen (1992), Nwogu (1993) and the extended mild-slope equations of Massel (1993), Suh et al. (1997), Lee et al. (1998), and Lee et al. (2003b). By applying the fractional step splitting method to the time-dependent wave equations including the source function, we found the equivalence of the two types of internal wave generation techniques, i.e., the source term addition method and the delta source function method. From the equivalent relation, we verified the energy velocity approach that the energy velocity should be used instead of the phase velocity for the transport of incident wave energy in the source term addition method. The source function method is verified numerically by generating accurately nonlinear cnoidal 
waves as well as linear waves for horizontally one-dimensional cases. All the numerical solutions show the target wave amplitudes are accurately generated by the source functions. While the Gaussian source function (Wei et al., 1999) needs several grids for generating waves accurately, the delta source function needs only one spatial grid which saves the storage and computational time. By the fractional step splitting method, the two wave generation methods are proved to be identical to each other. However, the delta source function method is recommended to be used rather than the source term addition method in terms of the mathematical completeness because the delta source function is included in the original wave equation and then the resulting equations are discretized numerically.

This energy velocity approach was also verified by Schäffer and Sørensen (2006). They added the delta source function to the mass conservation type equation, in tegrated asymptotically the resulting equation at the generation point, and thus they got an explicit relation of the source function to the energy velocity. However, they couldn't get the energy velocity for the extended Boussinesq equations of Madsen and Sørensen (1992) because the source function cannot be related explicitly to the energy velocity. To the contrary, we could get the energy velocities for all types of wave equations including the equations of Madsen and Sørensen.

In this study, we derived the source functions for the extended mild-slope equations, which are reduced to the mild-slope equations by excluding the terms of second-order bottom effects. Thus, the source functions of the extended models of Massel (1993), Suh et al. (1997), Lee et al. (1998), and Lee et al. (2003b) can be used to the models of Berkhoff (1972), Radder and Dingemans (1985), Copeland (1985), and Kubo et al. (1992), respectiv ely. 


\section{Appendix A. Green's function approach}

In order to obtain the relationship between the source function $s$ and the surface elevation $\eta$, a particular solution of the differential equation including the source function should be obtained. The differential equation after extracting both the $y$ directional and time-harmonic terms is given by

$C_{1} \frac{d^{4} \widetilde{\eta}}{d x^{4}}+C_{2} \frac{d^{2} \widetilde{\eta}}{d x^{2}}+C_{3} \widetilde{\eta}=C_{4} \widetilde{s}(x)$

For the conventional Boussinesq equations of Peregrine (1967) and all the extended mild-slope equations, $C_{1}=0, C_{2}=1$ and $C_{3}=l^{2}$, and thus the above equation becomes the Helmholtz equation. For the extended Boussinesq equations, $C_{1}, C_{2}$ and $C_{3}$ are listed in Eqs. (19a)-(19c) and Eqs. (28a) - (28c). $C_{4}$ is a coefficient of the source function. In order to obtain the particular solution for Eq. (73), we seek a Green's function $G(\xi, x)$ which satisfies the following equation

$C_{1} \frac{d^{4} G(\xi, x)}{d \xi^{4}}+C_{2} \frac{d^{2} G(\xi, x)}{d \xi^{2}}+C_{3} G(\xi, x)=\delta(\xi-x)$

where $\xi$ and $x$ are regarded as the active and fixed variables, respectively, and $\delta$ is 
the Dirac delta function. The variables of $G$ and $\tilde{\eta}$ should satisfy the radiation boundary conditions given by

$$
\begin{array}{llll}
\frac{d^{n} G}{d \xi^{n}}=(+i l)^{n} G, & \frac{d^{n} \widetilde{\eta}}{d \xi^{n}}=(+i l)^{n} \widetilde{\eta} & \text { as } & \xi \rightarrow+\infty \\
\frac{d^{n} G}{d \xi^{n}}=(-i l)^{n} G, & \frac{d^{n} \widetilde{\eta}}{d \xi^{n}}=(-i l)^{n} \tilde{\eta} & \text { as } & \xi \rightarrow-\infty
\end{array}
$$

\section{A.1 Boussinesq equations of Peregrine (1967)}

For Peregrine's Boussinesq equations, the coefficients of Eq. (73) are

$$
\begin{aligned}
& C_{1}=0, \quad C_{2}=1, \quad C_{3}=l^{2} \\
& C_{4}=i \frac{k^{2}}{\omega}\left\{1+\frac{(k h)^{2}}{3}\right\}
\end{aligned}
$$

Integrating Eq. (74) with respect to $\xi$ from $x-0$ to $x+0$ yields the following equation

$$
\left.\frac{d G}{d \xi}\right|_{\xi=x-0} ^{\xi=x+0}=1
$$

where we use the continuity condition of $G$ at $\xi=x$. The Green's function that satisfies Eq. (78) and the radiation conditions (75) and (76) is given by 
$G(\xi, x)= \begin{cases}G_{+}=A \exp \{i l(\xi-x)\}, & \xi>x \\ G_{-}=A \exp \{i l(x-\xi)\}, & \xi<x\end{cases}$

where the amplitude $A$ is

$$
A=-\frac{i}{2 l}
$$

Multiplying Eq. (74) by $\tilde{\eta}(\xi)$ and integrating with respect to $\xi$ from $-\infty$ to $+\infty$ give the following equation

$$
\int_{-\infty}^{+\infty}\left\{C_{1} \frac{d^{4} G(\xi, x)}{d \xi^{4}}+C_{2} \frac{d^{2} G(\xi, x)}{d \xi^{2}}+C_{3} G(\xi, x)\right\} \widetilde{\eta}(\xi) d \xi=\int_{-\infty}^{+\infty} \delta(\xi-x) \widetilde{\eta}(\xi) d \xi
$$

Integrating the above equation by parts and using the relation (73) and the radiation boundary conditions (75) and (76) give the following equation

$\tilde{\eta}(x)=C_{4}\left\{\int_{-\infty}^{x} G_{-}(\xi, x) \tilde{s}(\xi) d \xi+\int_{x}^{+\infty} G_{+}(\xi, x) \tilde{s}(\xi) d \xi\right\}$

For waves propagating in the positive $x$-direction, the second integral in the right side 
of Eq. (82) becomes zero in a concerned region $\left(x>x_{s}\right)$. Thus, Eq. (82) becomes

$\widetilde{\eta}(x)=C_{4} \int_{-\infty}^{x} G_{-}(\xi, x) \widetilde{s}(\xi) d \xi=C_{4} A D \exp \left\{i l\left(x-x_{s}\right)\right\}$

After using a similar way for waves propagating in the negative $x$-direction, we have the following solution

$\tilde{\eta}(x)=C_{4} A D \exp \left\{ \pm i l\left(x-x_{s}\right)\right\}$

where the upper plus and lower minus signs mean that waves are propagating in the right and left directions, respectively, from the source point of $x=x_{s}$. Using Eqs. (77d) and (80), we get the following solution

$\widetilde{\eta}=\frac{k^{2}}{2 \omega l}\left\{1+\frac{(k h)^{2}}{3}\right\} D \exp \left\{ \pm i l\left(x-x_{s}\right)\right\}$

\section{A.2 Extended Boussinesq equations of Madsen and Sørensen (1992)}

As the coefficient of Eq. (73) is $C_{1} \neq 0$ for the extended Boussinesq equations of Madsen and Sørensen, the Green's function which satisfies the differential equation (74) and the radiation conditions (75) and (76) is given by 
$G(\xi, x)= \begin{cases}G_{+}=A_{1} \exp \left\{i l_{1}(\xi-x)\right\}+A_{2} \exp \left\{i l_{2}(\xi-x)\right\}, & \xi>x \\ G_{-}=A_{1} \exp \left\{i l_{1}(x-\xi)\right\}+A_{2} \exp \left\{i l_{2}(x-\xi)\right\}, & \xi<x\end{cases}$

where the wave numbers $l_{1}$ and $l_{2}$ are

$l_{1}=\sqrt{\frac{C_{2}-\sqrt{C_{2}^{2}-4 C_{1} C_{3}}}{2 C_{1}}}, \quad l_{2}=\sqrt{\frac{C_{2}+\sqrt{C_{2}^{2}-4 C_{1} C_{3}}}{2 C_{1}}}$

where $l_{1}$ is the $x$-directional wave number of progressive waves and $l_{2}$ is the imaginary wave number of evanescent modes. As $G$ is continuous at $\xi=x$, we have

$l_{1} A_{1}+l_{2} A_{2}=0$

Integrating Eq. (74) and using the continuity conditions of $G, d G / d \xi$ and $d^{2} G / d \xi^{2}$ at $\xi=x$ yield the following equation

$\left.C_{1} \frac{d^{3} G}{d \xi^{3}}\right|_{\xi=x-0} ^{\xi=x+0}=1$

Thus, we have

$-2 C_{1} i\left(l_{1}^{3} A_{1}+l_{2}^{3} A_{2}\right)=1$

The amplitude $A_{1}$ can be obtained from Eqs. (88) and (90) as 
$A_{1}=\frac{i}{2 C_{1} l_{1}\left(l_{1}^{2}-l_{2}^{2}\right)}$

The dispersion relation for the extended Boussinesq equations of Madsen and Sorsensen is

$$
\frac{\omega}{k}=\sqrt{g h \frac{1+B(k h)^{2}}{1+(B+1 / 3)(k h)^{2}}}
$$

Using the dispersion relation of Eq. (92) and the definitions of $l_{1}$ and $l_{2}$, the amplitude $A_{1}$ can be expressed as

$$
A_{1}=-\frac{i k}{2\left(\omega^{2}+B g k^{4} h^{3}\right) \cos \theta}
$$

The amplitude $A_{2}$ of the evanescent mode with $l_{2}$ becomes negligible at a point away from the source point. Thus, we take into account only the propagating component with the amplitude $A_{1}$.

Multiplying Eq. (74) by $\tilde{\eta}(\xi)$ and integrating with respect to $\xi$ from $-\infty$ to $+\infty$ give Eq. (81). Then, integrating Eq. (81) by parts and using the relation (73) and the radiation boundary conditions (75) and (76) give the following equation 


$$
\begin{aligned}
\widetilde{\eta}(x)= & \int_{-\infty}^{+\infty} G(\xi, x) C_{4} \tilde{s} d \xi \\
= & i \omega\left\{\int_{-\infty}^{x} G_{-}(\xi, x)\left\{1-\left(B+\frac{1}{3}\right) h^{2}\left(\frac{d^{2}}{d x^{2}}-m^{2}\right)\right\} \tilde{s} d \xi\right. \\
& \left.+\int_{x}^{+\infty} G_{+}(\xi, x)\left\{1-\left(B+\frac{1}{3}\right) h^{2}\left(\frac{d^{2}}{d x^{2}}-m^{2}\right)\right\} \tilde{s} d \xi\right\}
\end{aligned}
$$

For waves propagating in the positive $x$-direction $\left(x>x_{s}\right)$, the above equation becomes

$$
\begin{aligned}
\widetilde{\eta}(x) & =i \omega \int_{-\infty}^{x} G_{-}(\xi, x)\left\{1-\left(B+\frac{1}{3}\right) h^{2}\left(\frac{d^{2}}{d x^{2}}-m^{2}\right)\right\} \tilde{s} d \xi \\
& =i \omega A_{1} D \int_{-\infty}^{x} \exp \{i l(x-\xi)\}\left\{1-\left(B+\frac{1}{3}\right) h^{2}\left(\frac{d^{2}}{d x^{2}}-m^{2}\right)\right\} \delta\left(\xi-x_{s}\right) d \xi \\
& =i \omega\left\{1+\left(B+\frac{1}{3}\right) k^{2} h^{2}\right\} A_{1} D \exp \left\{i l\left(x-x_{s}\right)\right\}
\end{aligned}
$$

where the integration of the second-derivative term of the delta function is obtained by integrating by parts as

$$
\int_{-\infty}^{x} \exp \{i l(x-\xi)\} \frac{d^{2} \delta\left(\xi-x_{s}\right)}{d x^{2}} d \xi=-l^{2} \exp \left\{i l\left(x-x_{s}\right)\right\}
$$

After following a similar way for waves propagating in the negative $x$-direction and then using Eq. (93), we have the following solution

$$
\widetilde{\eta}=\frac{\omega k\left\{1+\left(B+\frac{1}{3}\right)(k h)^{2}\right\}}{2\left\{\omega^{2}+B g k^{4} h^{3}\right\}} \frac{D}{\cos \theta} \exp \left\{ \pm i l\left(x-x_{s}\right)\right\}
$$




\section{A.3 Extended Boussinesq equations of Nwogu (1993)}

As the coefficient of Eq. (73) $C_{1} \neq 0$ for the extended Boussinesq equations of Nwogu, the Green's function which satisfies the differential equation (74) and the radiation conditions (75) and (76) is given by Eq. (86). To get the amplitude $A_{1}$, we follow the same procedure given in Eqs. (88) to (93) as for the extended Boussinesq equations of Madsen and Sørensen except using the dispersion relation given by

$$
\frac{\omega}{k}=\sqrt{g h \frac{1-(\alpha+1 / 3)(k h)^{2}}{1-\alpha(k h)^{2}}}
$$

Thus, we have the amplitude $A_{1}$ given by

$$
A_{1}=-\frac{i k}{2\left\{\omega^{2}-\left(\alpha+\frac{1}{3}\right) g k^{4} h^{3}\right\} \cos \theta}
$$

Then, to get the particular solution $\tilde{\eta}$, we follow the same procedure given in Eqs. (81) to (84) as for the Boussinesq equations of Peregrine except that the amplitude $A$ should be replaced by $A_{1}$ given in Eq. (99). Thus, using Eqs. (28d) and (99), we get the following solution

$$
\tilde{\eta}=\frac{\omega k\left\{1-\alpha(k h)^{2}\right\}}{2\left\{\omega^{2}-\left(\alpha+\frac{1}{3}\right) g k^{4} h^{3}\right\}} \frac{D}{\cos \theta} \exp \left\{ \pm i\left(x-x_{s}\right)\right\}
$$

\section{A.4 Extended mild-slope equations of Massel (1993), Suh et al. (1997), Lee et al. (1998), and Lee et al. (2003b)}


For extended mild-slope equation of Massel (1993), Suh et al. (1997), Lee et al. (1998), and Lee et al. (2003b) the coefficients of Eq. (73) are $C_{1}=0, C_{2}=1, C_{3}=l^{2}$, and

$$
\begin{array}{rlrl}
C_{4} & =i \frac{\omega}{g C C_{g}}, & & \text { Massel's (1993) equation } \\
C_{4} & =i \frac{\omega}{\bar{C} \bar{C}_{g}}, & & \text { Suh et al.'s (1997) equations } \\
C_{4} & =i \frac{k}{\bar{C}}, & & \text { Lee et al.'s (1998) equations } \\
C_{4}=\frac{1}{\bar{C} \bar{C}_{g}+\Delta \omega \frac{\partial\left(C C_{g}\right)}{\partial \omega}}, & \text { Lee et al.'s (2003b) equation }
\end{array}
$$

The Green's function which satisfies the differential equation (74) and the radiation conditions (75) and (76) is given by Eq. (79). Then, to get the particular solution $\widetilde{\eta}$, we follow the same procedure given in Eqs. (81) to (84) as for the Boussinesq equations of Peregrine. Thus, using Eqs. (80) and (101a)-(101d), we get the following solution

$$
\begin{array}{ll}
\tilde{\eta}(x)=\frac{1}{2 g C_{g} \cos \theta} D \exp \left\{ \pm i l\left(x-x_{s}\right)\right\}, & \text { Massel's (1993) equation } \\
\widetilde{\eta}=\frac{\omega}{2 l \bar{C} \bar{C}_{g}} D \exp \left\{ \pm i l\left(x-x_{s}\right)\right\}, & \text { Suh et al.'s (1997) equations } \\
\widetilde{\eta}=\frac{k}{2 l \bar{C}} D \exp \left\{ \pm i l\left(x-x_{s}\right)\right\}, & \text { Lee et al.'s (1998) equations } \\
\widetilde{\eta}=\frac{-i}{2 l\left\{\bar{C} \bar{C}_{g}+\Delta \omega \frac{\partial\left(C C_{g}\right)}{\partial \omega}\right\}} D \exp \left\{ \pm i l\left(x-x_{s}\right)\right\}, & \text { Lee et al.'s (2003b) equations }
\end{array}
$$

\section{Acknowledgements}

The second author was supported by the Basic Research Program of the Korea Science 
\& Engin eering Foundation (No.: R01-2003-000-10635-0). 


\section{References}

Bellotti, G., Beltrami, G.M., De Girolamo, P., 2003. Internal generation of waves in 2D fully ellip tic mild-slope equation FEM models. Coastal Eng. 49, 71-81.

Berkhoff, J.C.W., 1972. Computation of combined refraction-diffraction. Proc. 13th Coastal Eng. Conf., ASCE, 471-490.

Brorsen, M., Larsen, J. 1987. Source generation of nonlinear gravity waves with the boundary integral equation method. Coastal Eng. 11, 93-113.

Copeland, G.J.M., 1985. A practical alternative to the mild-slope wave equation. Coastal Eng. 9, 125-149.

Kim, G., Lee, C., Suh, K.-D., 2006. Generation of random waves in time-dependent extended mild-slope equations using a source function method. Ocean Eng. (in press).

Kirby, J.T., Lee, C., Rasmussen, C., 1992. Time-dependent solutions of the mild-slope wave equation. Proc. 23rd Int. Conf. on Coastal Eng., Venice, 391-404.

Kubo, Y., Kotake, Y., Isobe, M., Watanabe, A., 1992. Time-dependent mild slope equation for random waves. Proc. 23rd Int. Conf. Coastal Eng., ASCE, 419-431.

Larsen, J., Dancy, H., 1983. Open boundaries in short wave simulations - a new approach. Coastal Eng. 7, 285-297.

Lee, C., Cho, Y.-S. and Yoon, S.B., 2003a. A note on linear dispersion and shoaling properties in extended Boussinesq equations. Ocean Eng. 30, 1849-1867. 
Lee, C., Cho, Y.-S., Yum, K., 2001. Internal generation of waves for extended Boussin esq equations. Coastal Eng. 42, 155-162.

Lee, C., Kim, G., Suh, K.-D., 2003b. Extended mild-slope equation for random waves. Coastal Eng. 48, 277-287.

Lee, C., Park, W.S., Cho, Y.-S., Suh, K.D., 1998. Hyperbolic mild-slope equations extended to account for rapidly varying topography. Coastal Eng. 34, 243-257.

Lee, C., Suh, K.D., 1998. Internal generation of waves for time-dependent mild-slope equations. Coastal Eng. 34, 35-57.

Lee, C., Yoon, S.B., 2007. Internal generation of waves on an arc in a rectangular grid system. Coastal Eng. 54, 357-368.

LeVeque, R.J., 1998. Balancing source terms and flux gradients in high-resolution Godunov methods: the quasi-steady wave-propagation algorithm. J. of Computational Physics 146, 346-365.

Madsen, P.A., Larsen, J., 1987. An efficient finite-difference approach to the mild-slope equation. Coastal Eng. 11, 329-351.

Madsen, P.A., Murray, R., Sørensen, O.R., 1991. A new form of the Boussinesq equations with improved linear dispersion characteristics. Coastal Eng. 15, 371-388. 
Madsen, P.A., Sørensen, O.R. 1992. A new form of the Boussinesq equations with improved linear dispersion characteristic: part II. A slowly-varying bathymetry. Coastal Eng. 18, 183-204.

Massel, S.R., 1993. Extended refraction-diffraction equation for surface waves. Coastal Eng., 19, 97-126.

Nwogu, O., 1993. Alternative form of Boussinesq equations for nearshore wave propagation. J. Waterway, Port, Coastal and Ocean Eng. 119, 618-638.

Peregrine, D.H., 1967. Long waves on a beach. J. Fluid Mech. 27, 815-827.

Radder, A.C., Dingemans, M.W., 1985. Canonical equations for almost periodic, weakly nonlin ear gravity waves. Wave Motion 7, 473-485.

Schäffer, H.A., Sørensen, O.R., 2006. On the internal wave generation in Boussinesq and mild-slope equations. Coastal Eng. 53, 319-323.

Shapiro, R., 1970. Smoothing, filtering, and boundary effects. Review of Geophysics and Space Physics, 8(2), 359-386.

Suh, K.D., Lee, C., Park, W.S., 1997. Time-dependent equations for wave propagation on rapidly varying topography. Coastal Eng. 32, 91-117.

Wei, G., Kirby, J.T. 1995. Time-dependent numerical code for extended Boussinesq equations. J. Waterway, Port, Coastal and Ocean Eng. 121, 251-261. 
Wei, G., Kirby, J.T., Sinha, A., 1999. Generation of waves in Boussinesq models using a source function method. Coastal Eng. 36, 271-299. 


\section{Captions of Figures}

Figure 1. Computational domain.

Figure 2. Normalized amplitudes of linear waves versus relative water depth: (a) Peregrine (1967)'s conventional Boussinesq equations, (b) Madsen and Sørensen (1992)'s extended Boussinesq equations, (c) Nwogu (1993)'s extended Boussinesq equations, (d) Suh et al. (1997)'s extended mild-slope equations, (e) Lee et al. (1998)'s extended mild-slope equations, (f) Lee et al. (2003b)'s extended mild-slope equation.

Figure 3. Normalized water surface elevations and envelopes of linear waves for the extended mild-slope equations of Lee et al. (2003b): (a) $k_{e} h=0.1 \pi$, (b) $k_{e} h=\pi$; solid $\underline{\text { line }=\text { water surface elevation, thick vertical line }=\text { starting point of sponge layer. }}$

Figure 4. Normalized water surface elevations and envelopes of linear waves for the extended Boussinesq equations of Nwogu's (1993): (a) $k_{e} h=0.1 \pi$, (b) $k_{e} h=\pi$; $\underline{\text { solid }}$ $\underline{\text { line }=\text { water surface elevation, thick vertical line }=\text { starting point of sponge layer. }}$

Figure 5. Normalized water surface elevations and wave envelopes of cnoidal waves for the Boussinesq equations of Peregrine (1967): (a) $|\eta|^{I}=1 \mathrm{~m}$, (b) $|\eta|^{I}=2 \mathrm{~m}$, (c) $|\eta|^{I}=3$ m.; solid line $=$ numerical solution of water surface elevation, dashed line $=$ analytical solution of water surface elevation, dash-dotted line $=$ numerical solution of wave envelope, thick vertical line $=$ starting point of sponge layer. 


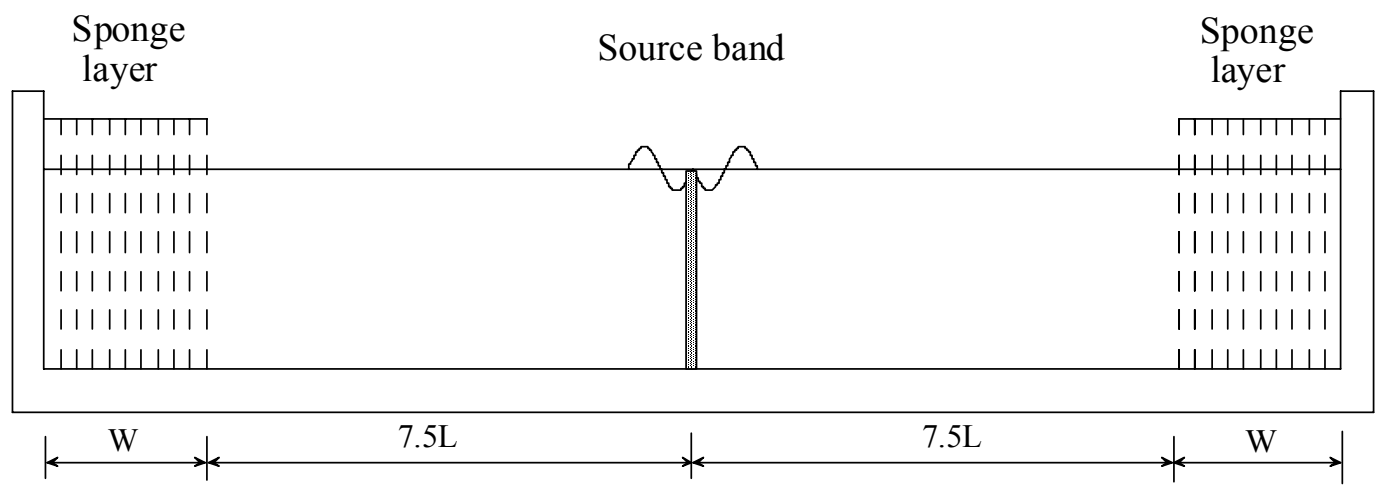

Figure 1. Computational domain.

Figure 1 
(a)

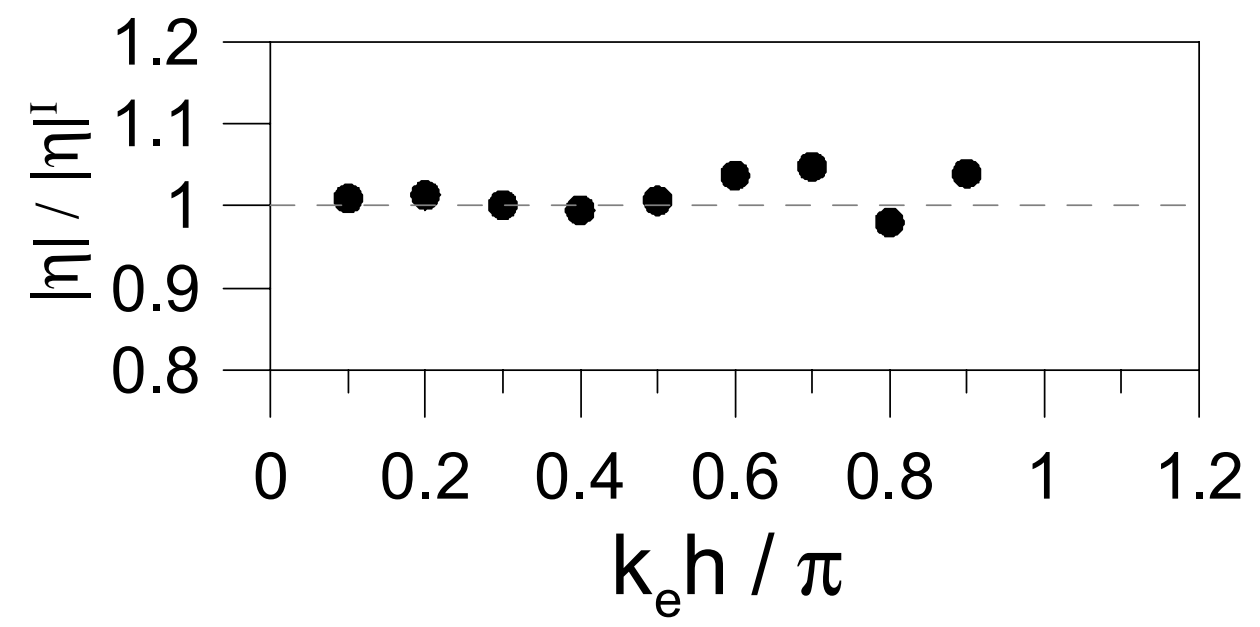

(b)

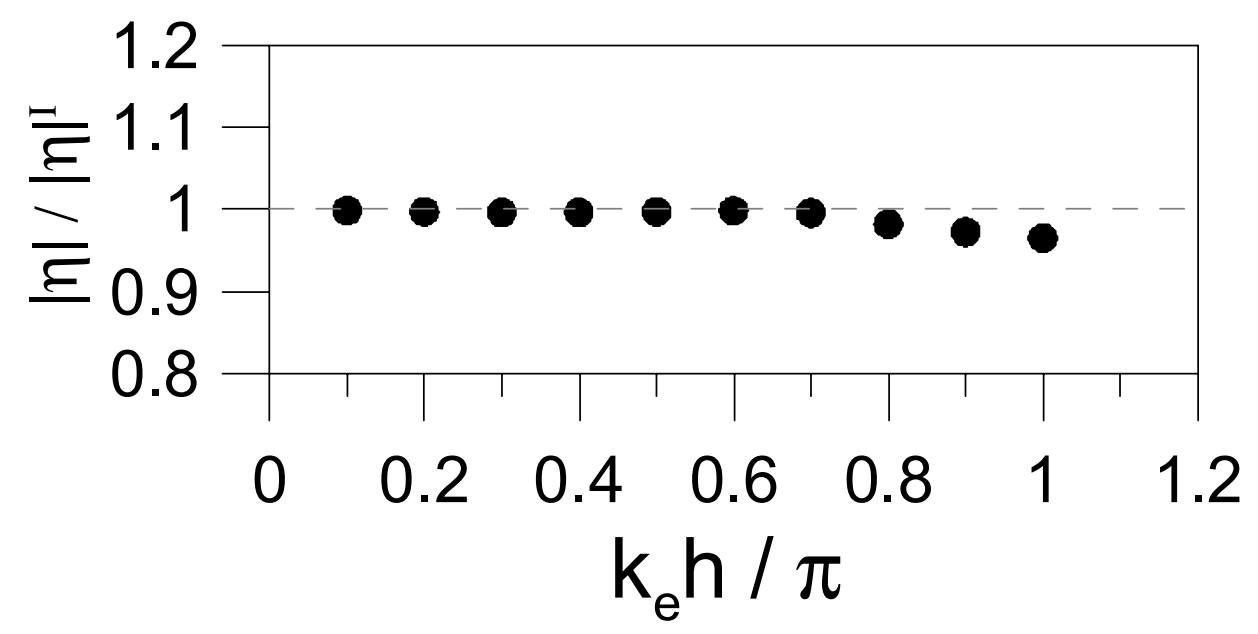

Figure 2. Normalized amplitudes of linear waves versus relative water depth: (a) Peregrine (1967)'s conventional Boussinesq equations, (b) Madsen and Sørensen (1992)'s extended Boussinesq equations, (c) Nwogu (1993)'s extended Boussinesq equations, (d) Suh et al. (1997)'s extended mild-slope equations, (e) Lee et al. (1998)'s extended mild-slope equations, (f) Lee et al. (2003b)'s extended mild-slope equation. 
(c)

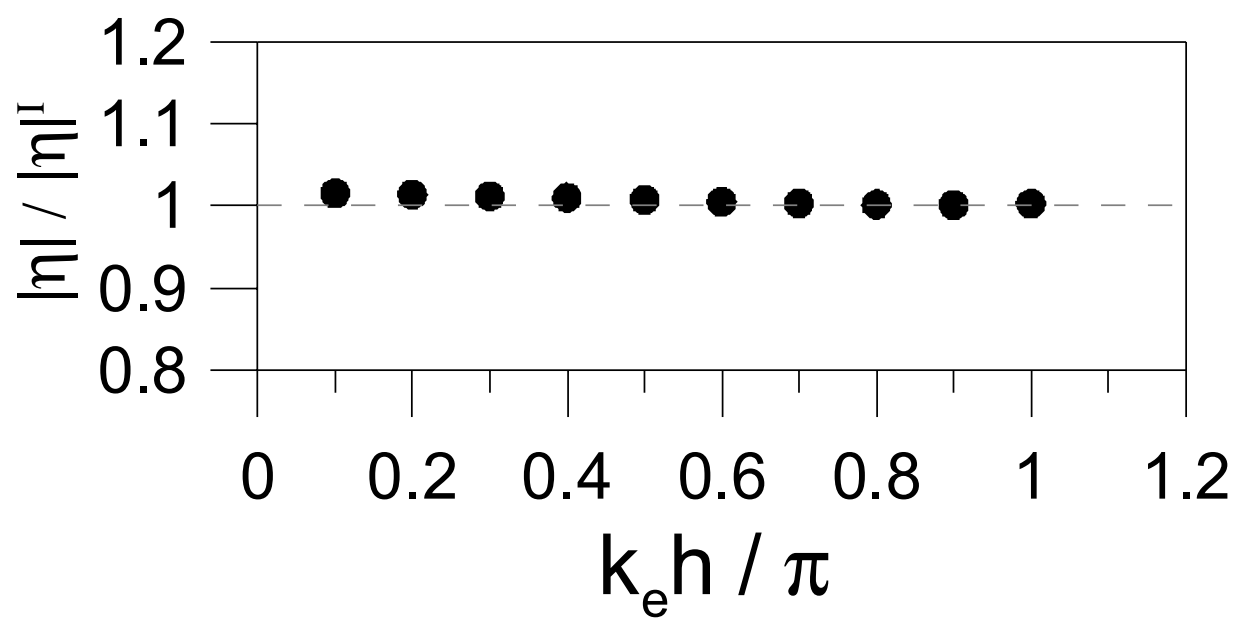

(d)

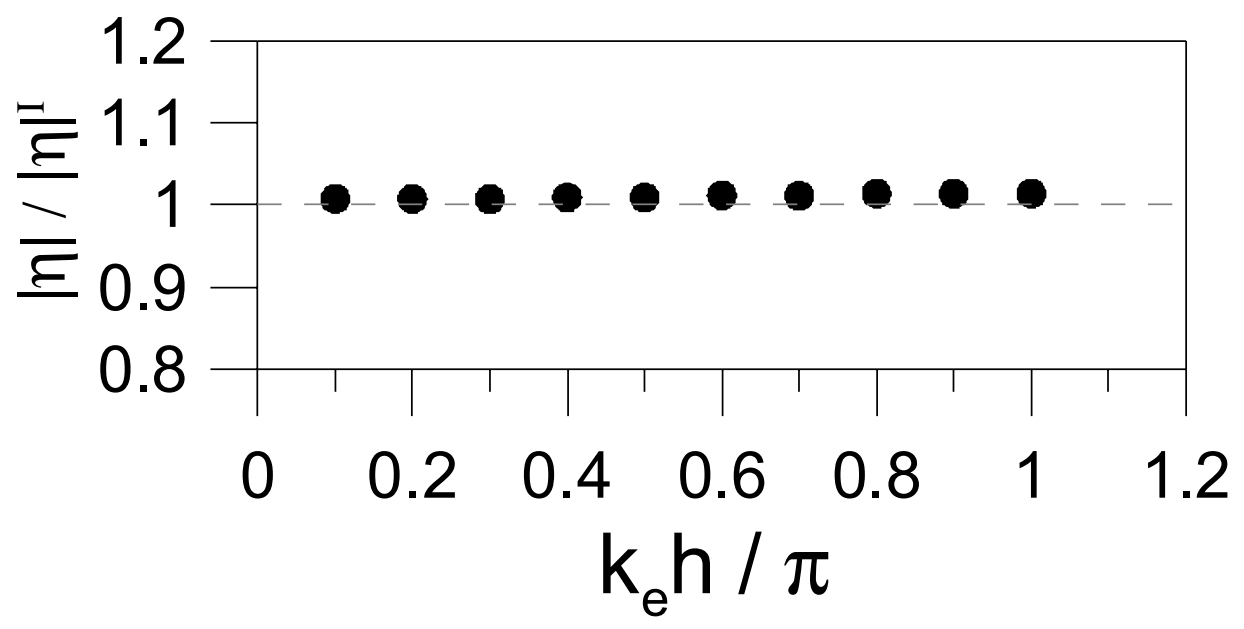

Figure 2. (Continued). 
(e)

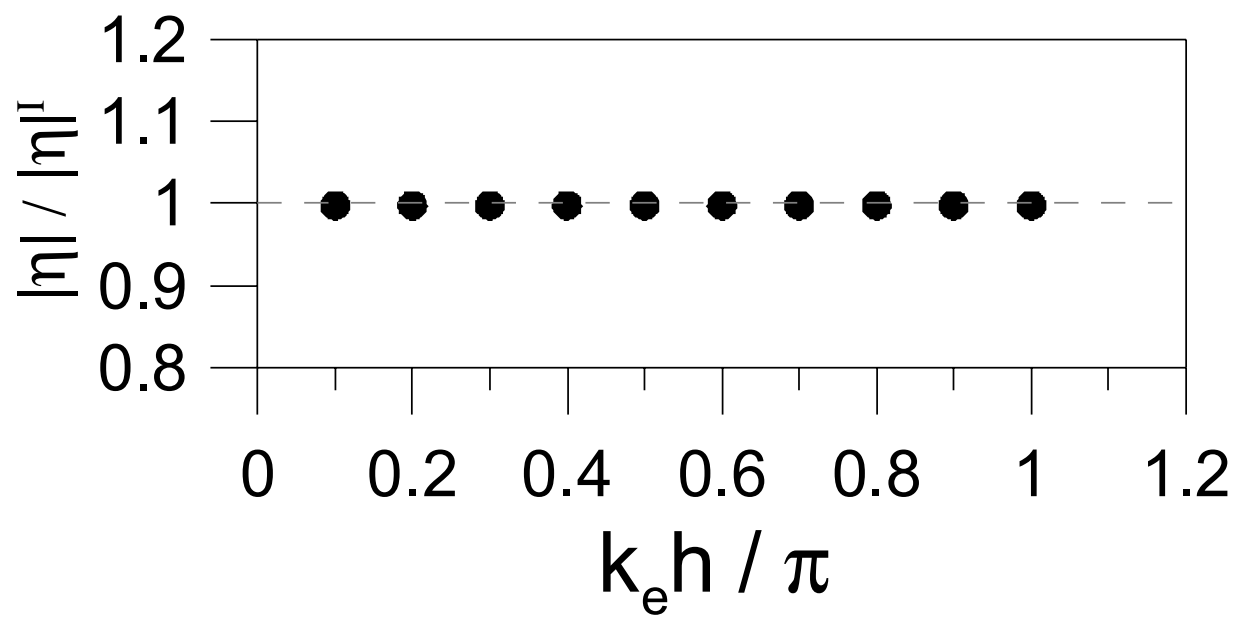

(f)

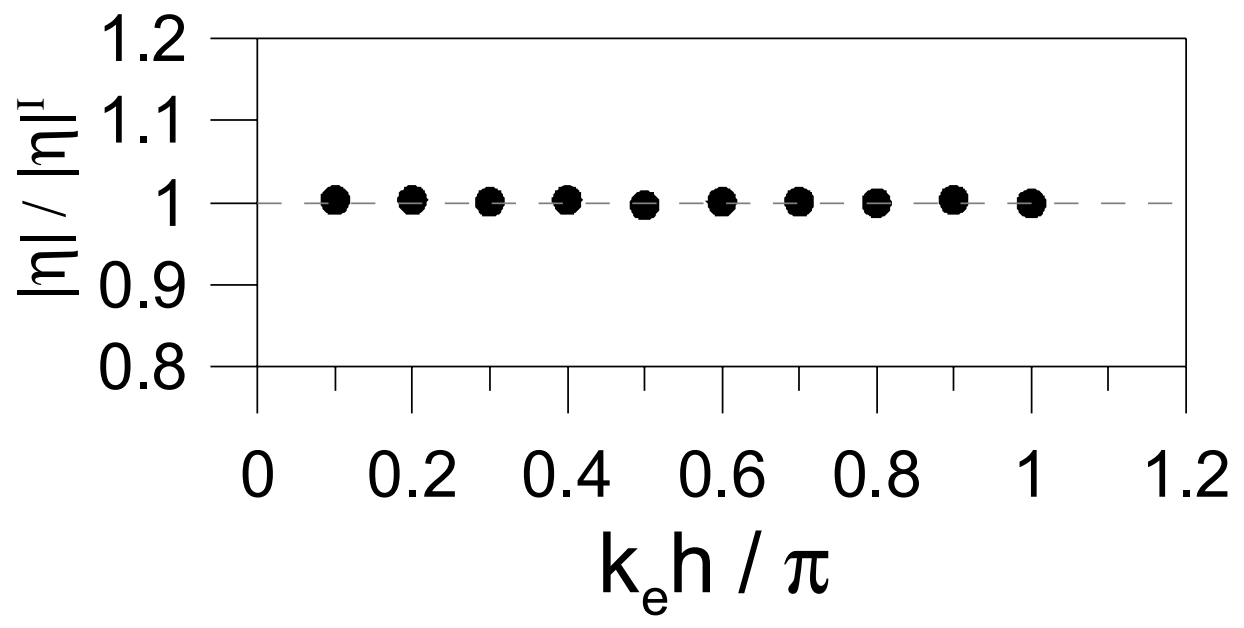

Figure 2. (Continued). 
(a)

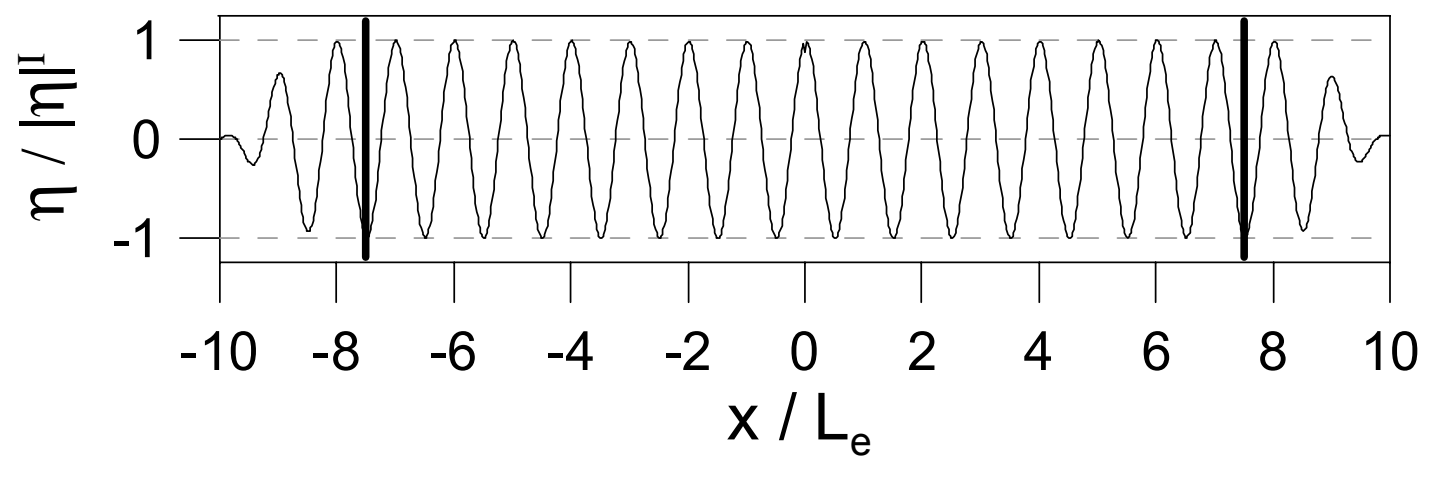

(b)

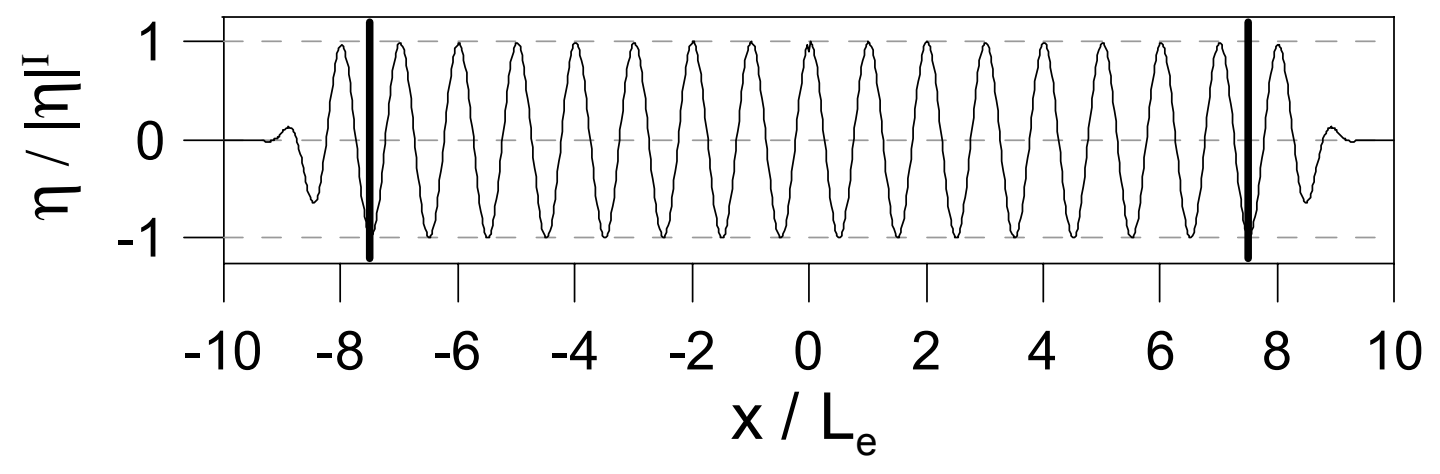

Figure 3. Normalized water surface elevations and envelopes of linear waves for the extended mild-slope equations of Lee et al. (2003b): (a) $k_{e} h=0.1 \pi$, (b) $k_{e} h=\pi$; solid $\underline{\text { line }=\text { water surface elevation, thick vertical line }=\text { starting point of sponge layer. }}$ 
(a)

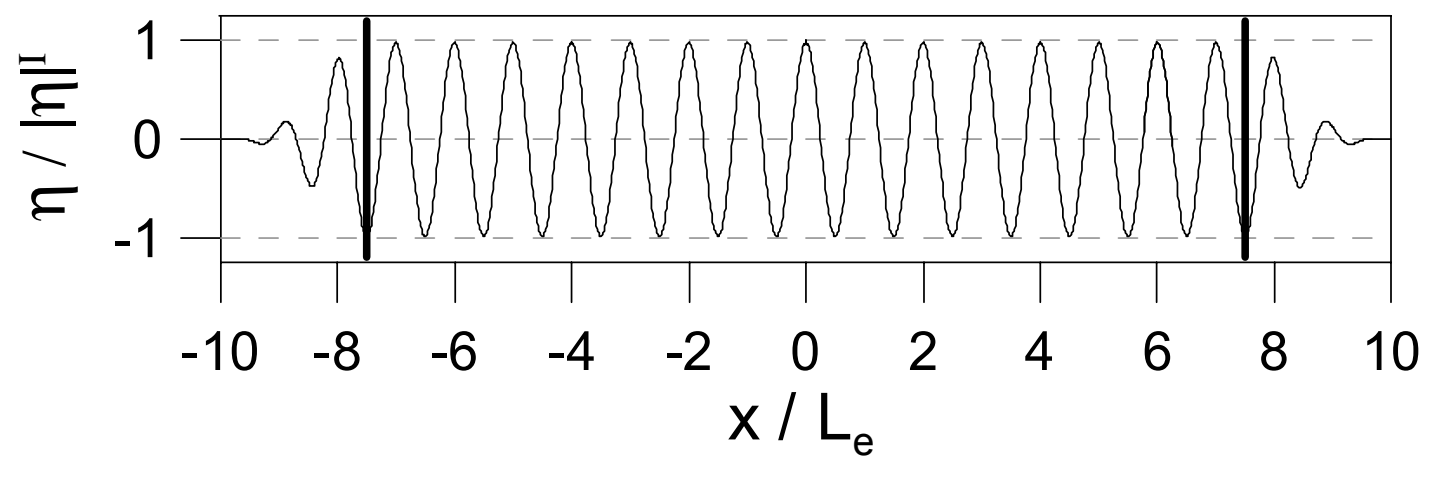

(b)

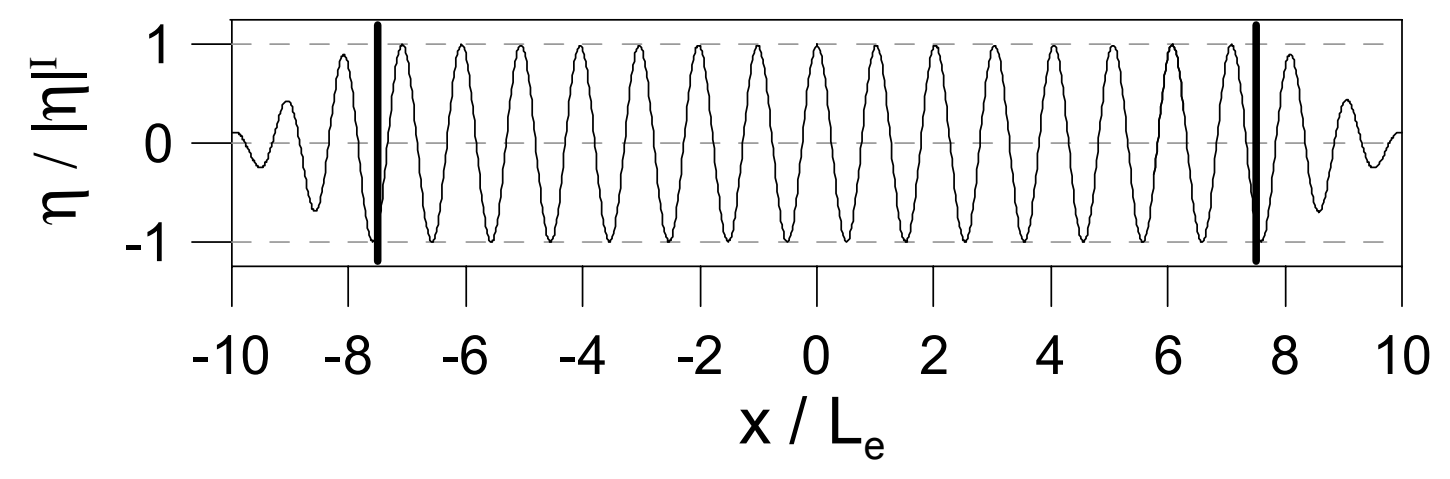

Figure 4. Normalized water surface elevations and envelopes of linear waves for the extended Boussinesq equations of Nwogu's (1993): (a) $k_{e} h=0.1 \pi$, (b) $k_{e} h=\pi$; solid $\underline{\text { line }=\text { water surface elevation, thick vertical line }=\text { starting point of sponge layer. }}$ 
(a)

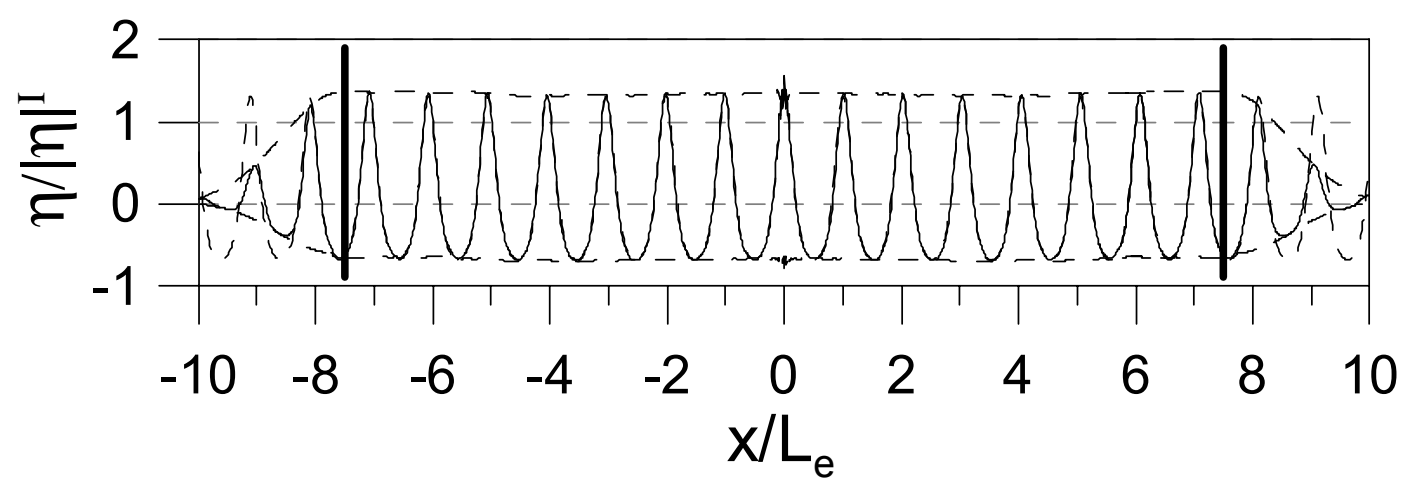

(b)

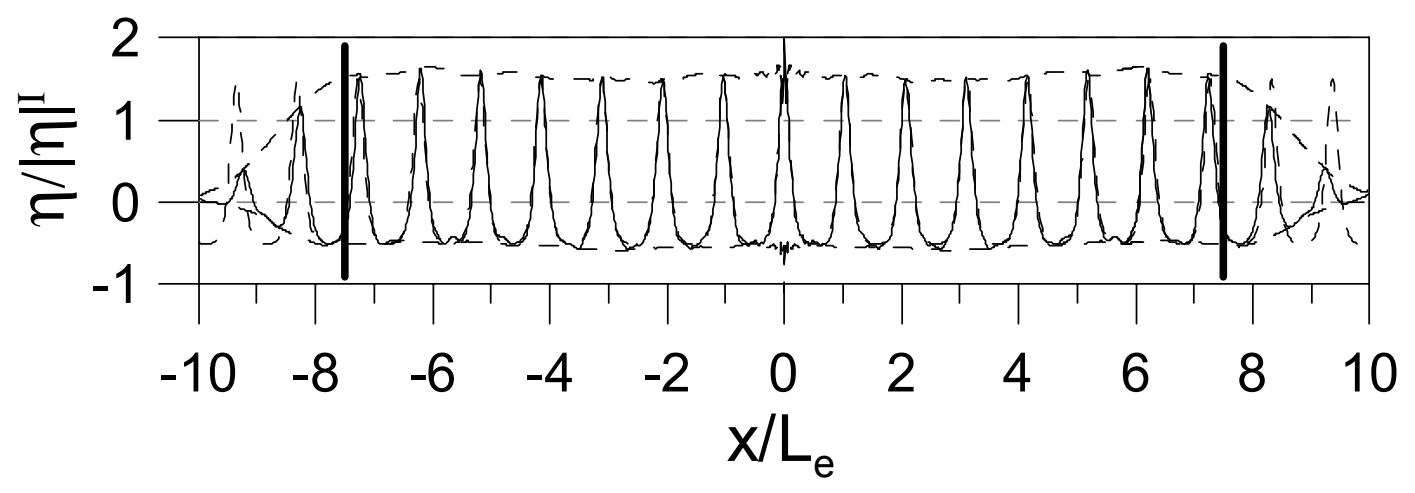

(c)

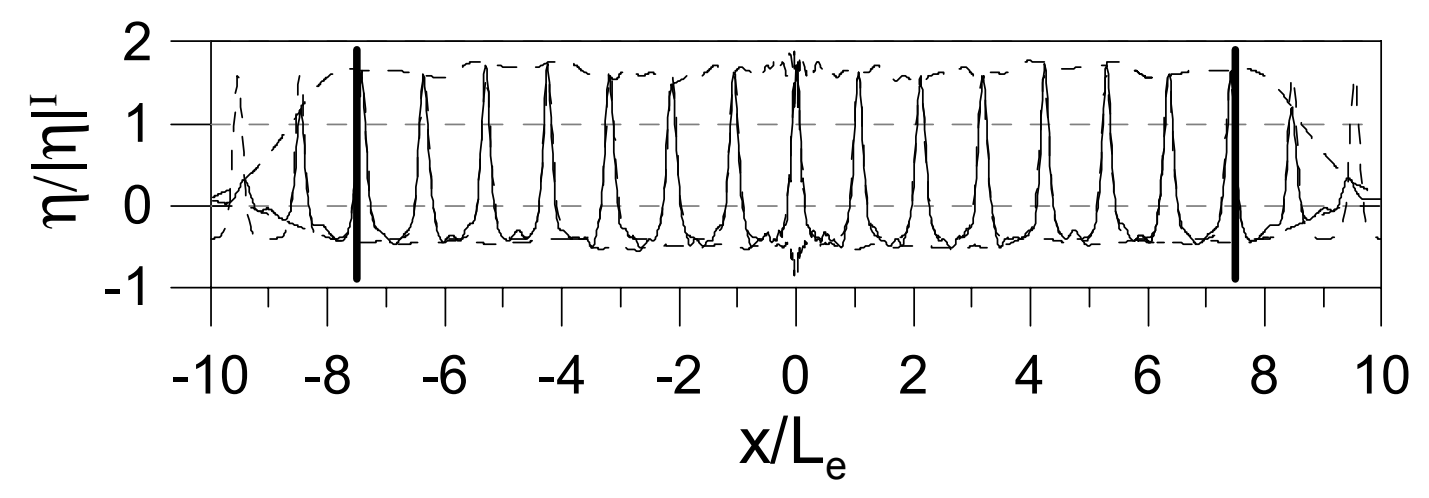

Figure 5. Normalized water surface elevations and wave envelopes of cnoidal waves for the Boussinesq equations of Peregrine (1967): (a) $|\eta|^{I}=1 \mathrm{~m}$, (b) $|\eta|^{I}=2 \mathrm{~m}$, (c) $|\eta|^{I}=3$ $\mathrm{m}$.; solid line $=$ numerical solution of water surface elevation, dashed line $=$ analytical solution of water surface elevation, dash-dotted line $=$ numerical solution of wave envelope, thick vertical line $=$ starting point of sponge layer. 